JOURNAL OF THE

AMERICAN MATHEMATICAL SOCIETY

Volume 11, Number 3, July 1998, Pages 569-600

S 0894-0347(98)00261-6

\title{
RELATIVE BOGOMOLOV'S INEQUALITY AND THE CONE OF POSITIVE DIVISORS ON THE MODULI SPACE OF STABLE CURVES
}

\author{
ATSUSHI MORIWAKI
}

\section{INTRODUCTION}

Throughout this paper, we fix an algebraically closed field $k$.

Let $f: X \rightarrow Y$ be a surjective and projective morphism of quasi-projective varieties over $k$ with $\operatorname{dim} f=1$. Let $E$ be a vector bundle of rank $r$ on $X$. Then, we define the discriminant divisor of $E$ with respect to $f: X \rightarrow Y$ to be

$$
\operatorname{dis}_{X / Y}(E)=f_{*}\left(\left(2 r c_{2}(E)-(r-1) c_{1}(E)^{2}\right) \cap[X]\right) .
$$

Here $f_{*}$ is the push-forward of cycles, so that $\operatorname{dis}_{X / Y}(E)$ is a divisor modulo linear equivalence on $Y$. In this paper, we would like to show the following theorem (cf. Corollary 2.5) and give its applications.

Theorem A $(\operatorname{char}(k)=0)$. We assume that $Y$ is smooth over $k$. Let $y$ be a point of $Y$, and let $\overline{\kappa(y)}$ be the algebraic closure of the residue field $\kappa(y)$ at $y$. If $f$ is flat over $y$, the geometric fiber $X_{\bar{y}}=X \times_{Y} \operatorname{Spec}(\overline{\kappa(y)})$ over $y$ is reduced and Gorenstein, and $E$ is semistable on each connected component of the normalization of $X_{\bar{y}}$, then $\operatorname{dis}_{X / Y}(E)$ is weakly positive at $y$, namely, for any ample divisors $A$ on $Y$ and any positive integers $n$, there is a positive integer $m$ such that

$$
H^{0}\left(Y, \mathcal{O}_{Y}\left(m\left(n \operatorname{dis}_{X / Y}(E)+A\right)\right)\right) \otimes \mathcal{O}_{Y} \rightarrow \mathcal{O}_{Y}\left(m\left(n \operatorname{dis}_{X / Y}(E)+A\right)\right)
$$

is surjective at $y$. Note that this theorem still holds in positive characteristic under the strong semistability of $E_{\bar{y}}$ (cf. Corollary 7.4$)$.

An interesting point of the above theorem is that even if the weak positivity of $\operatorname{dis}_{X / Y}(E)$ at $y$ is a global property on $Y$, it can be derived from the local assumption "the goodness of $X_{\bar{y}}$ and the semistability of $E_{\bar{y}}$ ". This gives a great advantage to our applications.

In order to understand the intuition underlying the theorem, let us consider a simulated case. Namely, we suppose that $f: X \rightarrow Y$ is a smooth surface fibred over a curve and the fiber is general. Bogomolov's instability theorem [1] says that if $E_{\bar{y}}$ is semistable, then the codimension two cycle $2 r c_{2}(E)-(r-1) c_{1}(E)^{2}$ has nonnegative degree. So if we push it down to a codimension one cycle on $Y$, then one can rephrase Bogomolov's theorem as saying that the semistability of $E_{y}$ implies the non-negativity of $\operatorname{dis}_{X / Y}(E)$.

Received by the editors April 17, 1997 and, in revised form, January 2, 1998.

1991 Mathematics Subject Classification. Primary 14H10, 14C20; Secondary 14G40.

Key words and phrases. Bogomolov's inequality, moduli space, stable curve. 
An immediate application of our inequality is a solution concerning the positivity of divisors on the moduli space of stable curves. Let $g \geq 2$ be an integer, and let $\overline{\mathcal{M}}_{g}$ (resp. $\mathcal{M}_{g}$ ) be the moduli space of stable (resp. smooth) curves of genus $g$ over $k$. The boundary $\overline{\mathcal{M}}_{g} \backslash \mathcal{M}_{g}$ is of codimension one and has $[g / 2]+1$ irreducible components, say, $\Delta_{0}, \Delta_{1}, \ldots, \Delta_{[g / 2]}$. The geometrical meaning of index is as follows. A general point of $\Delta_{0}$ represents an irreducible stable curve with one node, and a general point of $\Delta_{i}(i>0)$ represents a stable curve consisting of a curve of genus $i$ and a curve of genus $g-i$ joined at one point. Let $\delta_{i}$ be the class of $\Delta_{i}$ in $\operatorname{Pic}\left(\overline{\mathcal{M}}_{g}\right) \otimes \mathbb{Q}\left(\right.$ strictly speaking, $\delta_{i}=c_{1}\left(\mathcal{O}\left(\Delta_{i}\right)\right)$ for $i \neq 1$, and $\delta_{1}=\frac{1}{2} c_{1}\left(\mathcal{O}\left(\Delta_{1}\right)\right)$ ), and let $\lambda$ be the Hodge class on $\overline{\mathcal{M}}_{g}$. A fundamental problem due to Mumford [17] is to decide which $\mathbb{Q}$-divisor

$$
a \lambda-b_{0} \delta_{0}-b_{1} \delta_{1}-\cdots-b_{[g / 2]} \delta_{[g / 2]}
$$

is positive, where $a, b_{0}, \ldots, b_{[g / 2]}$ are rational numbers. Here, we can use a lot of types of positivity, namely, ampleness, numerical effectivity, effectivity, pseudoeffectivity, and so on. Besides them, we would like to introduce a new sort of positivity for our purposes. Let $V$ be a projective variety over $k$ and $U$ a nonempty Zariski open set of $V$. A $\mathbb{Q}$-Cartier divisor $D$ on $V$ is said to be numerically effective over $U$ if $(D \cdot C) \geq 0$ for all irreducible curves $C$ on $V$ with $C \cap U \neq \emptyset$. A first general result in this direction was found by Cornalba-Harris [3], Xiao [20] and Bost [2]. They proved that the $\mathbb{Q}$-divisor

$$
(8 g+4) \lambda-g\left(\delta_{0}+\delta_{1}+\cdots+\delta_{[g / 2]}\right)
$$

is numerically effective over $\mathcal{M}_{g}$. As we observed in [15] and [16], it is not sharp in coefficients of $\delta_{i}(i>0)$. Actually, the existence of a certain refinement of the above result was predicted at the end of the paper [3]. Our solution for this problem is the following (cf. Theorem 3.2 and Proposition 1.7).

Theorem B $(\operatorname{char}(k)=0)$. The divisor

$$
(8 g+4) \lambda-g \delta_{0}-\sum_{i=1}^{[g / 2]} 4 i(g-i) \delta_{i}
$$

is weakly positive over $\mathcal{M}_{g}$, i.e., if we denote the above divisor by $D$, then for any ample $\mathbb{Q}$-Cartier divisors $A$ on $\overline{\mathcal{M}}_{g}$, there is a positive integer $n$ such that $n(D+A)$ is a Cartier divisor and

$$
H^{0}\left(\overline{\mathcal{M}}_{g}, \mathcal{O}_{\overline{\mathcal{M}}_{g}}(n(D+A))\right) \otimes \mathcal{O}_{\overline{\mathcal{M}}_{g}} \rightarrow \mathcal{O}_{\overline{\mathcal{M}}_{g}}(n(D+A))
$$

is surjective on $\mathcal{M}_{g}$. In particular, it is pseudo-effective, and numerically effective over $\mathcal{M}_{g}$.

As an application of this theorem, we can decide the cone of weakly positive divisors over $\mathcal{M}_{g}$ (cf. Corollary 4.4).

Theorem $\mathbf{C}(\operatorname{char}(k)=0)$. If we denote by $\mathrm{WP}\left(\overline{\mathcal{M}}_{g} ; \mathcal{M}_{g}\right)$ the cone in $\operatorname{Pic}\left(\overline{\mathcal{M}}_{g}\right) \otimes$ $\mathbb{Q}$ consisting of weakly positive $\mathbb{Q}$-Cartier divisors over $\mathcal{M}_{g}$, then

$$
\mathrm{WP}\left(\overline{\mathcal{M}}_{g} ; \mathcal{M}_{g}\right)=\left\{\begin{array}{l|l}
x \lambda+\sum_{i=0}^{[g / 2]} y_{i} \delta_{i} & \begin{array}{l}
x \geq 0 \\
g x+(8 g+4) y_{0} \geq 0, \\
i(g-i) x+(2 g+1) y_{i} \geq 0 \quad(1 \leq i \leq[g / 2])
\end{array}
\end{array}\right\} .
$$


Moreover, using Theorem B, we can deduce a certain kind of inequality on an algebraic surface. In order to give an exact statement, we will introduce types of nodes of semistable curves. Let $Z$ be a semistable curve over $k$, and $P$ a node of $Z$. We can assign a number $i$ to the node $P$ in the following way. Let $\iota_{P}: Z_{P} \rightarrow Z$ be the partial normalization of $Z$ at $P$. If $Z_{P}$ is connected, then $i=0$. Otherwise, $i$ is the minimum of arithmetic genera of two connected components of $Z_{P}$. We say the node $P$ of $Z$ is of type $i$.

Let $X$ be a smooth projective surface over $k, Y$ a smooth projective curve over $k$, and $f: X \rightarrow Y$ a semistable curve of genus $g \geq 2$ over $Y$. By abuse of notation, we denote by $\delta_{i}(X / Y)$ the number of nodes of type $i$ in all singular fibers of $f$. Actually, $\delta_{i}(X / Y)=\operatorname{deg}\left(\pi^{*}\left(\delta_{i}\right)\right)$, where $\pi: Y \rightarrow \overline{\mathcal{M}}_{g}$ is the morphism induced by $f: X \rightarrow Y$. Then, we have the following (cf. Corollary 3.3).

Theorem $\mathbf{D}(\operatorname{char}(k)=0)$. With notation as above, we have the inequality

$$
(8 g+4) \operatorname{deg}\left(f_{*}\left(\omega_{X / Y}\right)\right) \geq g \delta_{0}(X / Y)+\sum_{i=1}^{[g / 2]} 4 i(g-i) \delta_{i}(X / Y) .
$$

As an arithmetic application of Theorem D, we can show the following answer for the effective Bogomolov's conjecture over function fields (cf. Theorem 5.2). (Recently, Bogomolov's conjecture over number fields was solved by Ullmo [19], but the effective Bogomolov's conjecture is still open.)

Theorem $\mathbf{E}(\operatorname{char}(k)=0)$. We assume that $f$ is not smooth and every singular fiber of $f$ is a tree of stable components, i.e., every node of type 0 on the stable model of each singular fiber is a singularity of an irreducible component. Then the effective Bogomolov's conjecture holds for the generic fiber of $f$. Namely, let $K$ be the function field of $Y, C$ the generic fiber of $f, \operatorname{Jac}(C)$ the Jacobian of $C$, and let $j: C(\bar{K}) \rightarrow \operatorname{Jac}(C)(\bar{K})$ be the morphism given by $j(x)=(2 g-2) x-\omega_{C}$. Then, the set $\left\{x \in C(\bar{K}) \mid\|j(x)-P\|_{N T} \leq r\right\}$ is finite for any $P \in \operatorname{Jac}(C)(\bar{K})$ and any non-negative real numbers $r$ less than

$$
\sqrt{\frac{(g-1)^{2}}{g(2 g+1)}\left(\frac{g-1}{3} \delta_{0}(X / Y)+\sum_{i=1}^{\left[\frac{g}{2}\right]} 4 i(g-i) \delta_{i}(X / Y)\right)}
$$

where \|\|$_{N T}$ is the semi-norm arising from the Neron-Tate height pairing on $\operatorname{Jac}(C)(\bar{K})$.

Finally, we would like to express our hearty thanks to the Institut des Hautes Études Scientifiques where all the work on this paper was done, and to Prof. Bost who pointed out a fatal error in the previous version. We are also grateful to the referees for their wonderful suggestions.

\section{ElEMENTARY PROPERTIES OF SEMI-AMPLENESS AND WEAK POSITIVITY}

In this section, we will introduce two kinds of positivity of divisors, namely semi-ampleness and weak positivity, and investigate their elementary properties.

Let $X$ be a $d$-dimensional algebraic variety over $k$. Let $Z_{d-1}(X)$ be a free abelian group generated by integral subvarieties of dimension $d-1$, and let $\operatorname{Div}(X)$ be a group consisting of Cartier divisors on $X$. We denote $Z_{d-1}(X)(\operatorname{resp} . \operatorname{Div}(X))$ 
modulo linear equivalence by $A_{d-1}(X)(\operatorname{resp}$. Pic $(X))$. An element of $Z_{d-1}(X) \otimes \mathbb{Q}$ $($ resp. $\operatorname{Div}(X) \otimes \mathbb{Q})$ is called a $\mathbb{Q}$-divisor (resp. $\mathbb{Q}$-Cartier divisor) on $X$.

We say a $\mathbb{Q}$-Cartier divisor $D$ is the limit of a sequence $\left\{D_{m}\right\}_{m=1}^{\infty}$ of $\mathbb{Q}$-Cartier divisors in $\operatorname{Pic}(X) \otimes \mathbb{Q}$, denoted by $D=\lim _{m \rightarrow \infty} D_{m}$ in $\operatorname{Pic}(X) \otimes \mathbb{Q}$, if there are $\mathbb{Q}$-Cartier divisors $Z_{1}, \ldots, Z_{l}$ and infinite sequences $\left\{a_{1, m}\right\}_{m=1}^{\infty}, \ldots,\left\{a_{l, m}\right\}_{m=1}^{\infty}$ of rational numbers such that (1) $l$ does not depend on $m$, (2) $D=D_{m}+\sum_{i=1}^{l} a_{i, m} Z_{i}$ in $\operatorname{Pic}(X) \otimes \mathbb{Q}$ for all $m \geq 1$, and (3) $\lim _{m \rightarrow \infty} a_{i, m}=0$ for all $i=1, \ldots, l$. For example, a pseudo-effective $\mathbb{Q}$-Cartier divisor is the limit of effective $\mathbb{Q}$-Cartier divisors in $\operatorname{Pic}(X) \otimes \mathbb{Q}$.

Let $x$ be a point of $X$. A $\mathbb{Q}$-Cartier divisor $D$ on $X$ is said to be semi-ample at $x$ if there is a positive integer $n$ such that $n D \in \operatorname{Div}(X)$ and $H^{0}\left(X, \mathcal{O}_{X}(n D)\right) \otimes \mathcal{O}_{X} \rightarrow$ $\mathcal{O}_{X}(n D)$ is surjective at $x$. Further, according to Viehweg, $D$ is said to be weakly positive at $x$ if there is an infinite sequence $\left\{D_{m}\right\}_{m=1}^{\infty}$ of $\mathbb{Q}$-Cartier divisors on $X$ such that $D_{m}$ is semi-ample at $x$ for all $m \geq 1$ and $D=\lim _{m \rightarrow \infty} D_{m}$ in $\operatorname{Pic}(X) \otimes \mathbb{Q}$. It is easy to see that if $D$ is weakly positive at $x$, then $(D \cdot C) \stackrel{m}{\rightarrow \infty} \geq 0$ for any complete irreducible curves $C$ passing through $x$. As compared with the last property, weak positivity has the advantage that we can avoid bad subvarieties of codimension two (cf. Proposition 1.4).

In order to consider properties of semi-ample or weakly positive divisors, let us begin with the following two lemmas.

Lemma $1.1(\operatorname{char}(k) \geq 0)$. Let $\pi: X \rightarrow Y$ be a proper morphism of quasi-projective varieties over $k$, and let $y$ be a point of $Y$ such that $\pi$ is finite over $y$. Let $F$ be a coherent $\mathcal{O}_{X}$-module and $H$ an ample line bundle on $Y$. Then there is a positive integer $n_{0}$ such that, for all $n \geq n_{0}$,

$$
H^{0}\left(X, F \otimes \pi^{*}\left(H^{\otimes n}\right)\right) \otimes \mathcal{O}_{X} \rightarrow F \otimes \pi^{*}\left(H^{\otimes n}\right)
$$

is surjective at each point of $\pi^{-1}(y)$.

Proof. Let $n_{0}$ be a positive integer such that, for all $n \geq n_{0}, \pi_{*}(F) \otimes H^{\otimes n}$ is generated by global sections, i.e.,

$$
H^{0}\left(Y, \pi_{*}(F) \otimes H^{\otimes n}\right) \otimes \mathcal{O}_{Y} \rightarrow \pi_{*}(F) \otimes H^{\otimes n}
$$

is surjective. Thus,

$$
H^{0}\left(X, F \otimes \pi^{*}\left(H^{\otimes n}\right)\right) \otimes \mathcal{O}_{X} \rightarrow \pi^{*} \pi_{*}\left(F \otimes \pi^{*}\left(H^{\otimes n}\right)\right)
$$

is surjective because $\pi_{*}\left(F \otimes \pi^{*}\left(H^{\otimes n}\right)\right)=\pi_{*}(F) \otimes H^{\otimes n}$. On the other hand, since $\pi$ is finite over $y$,

$$
\pi^{*} \pi_{*}\left(F \otimes \pi^{*}\left(H^{\otimes n}\right)\right) \rightarrow F \otimes \pi^{*}\left(H^{\otimes n}\right)
$$

is surjective at each point of $\pi^{-1}(y)$. Thus, we get our assertion.

Lemma $1.2(\operatorname{char}(k) \geq 0)$. Let $\pi: X \rightarrow Y$ be a proper morphism of quasi-projective varieties over $k$, and let $x$ be a point of $X$ such that $\pi$ is finite over $\pi(x)$. Let $D$ be a $\mathbb{Q}$-Cartier divisor on $X$ and $A$ a $\mathbb{Q}$-Cartier divisor on $Y$. If $D$ is weakly positive at $x$ and $A$ is ample, then $D+\pi^{*}(A)$ is semi-ample at $x$.

Proof. By our assumption, there are $\mathbb{Q}$-Cartier divisors $Z_{1}, \ldots, Z_{l}$, an infinite sequence $\left\{D_{m}\right\}_{m=1}^{\infty}$ of $\mathbb{Q}$-Cartier divisors, and infinite sequences $\left\{a_{1, m}\right\}_{m=1}^{\infty}, \ldots$, 
$\left\{a_{l, m}\right\}_{m=1}^{\infty}$ of rational numbers with the following properties:

(i) $D=D_{m}+\sum_{i=1}^{l} a_{i, m} Z_{i}$ in $\operatorname{Pic}(X) \otimes \mathbb{Q}$ for sufficiently large $m$.

(ii) $\lim _{m \rightarrow \infty} a_{i, m}=0$ for all $i=1, \ldots, l$.

(iii) $D_{m}$ is semi-ample at $x$ for $m \gg 0$.

By virtue of Lemma 1.1, we can find a positive integer $n$ such that $n \pi^{*}(A)+Z_{i}$ and $n \pi^{*}(A)-Z_{i}$ are semi-ample at $x$ for all $i$. We choose a sufficiently large $m$ with $\left|n l a_{i, m}\right|<1(i=1, \ldots, l)$. Then,

$$
\frac{1+n l a_{i, m}}{2 l n}>0 \text { and } \quad \frac{1-n l a_{i, m}}{2 l n}>0 .
$$

On the other hand,

$$
D+\pi^{*}(A) \sim D_{m}+\sum_{i=1}^{l}\left(\frac{1+n l a_{i, m}}{2 l n}\left(n \pi^{*}(A)+Z_{i}\right)+\frac{1-n l a_{i, m}}{2 l n}\left(n \pi^{*}(A)-Z_{i}\right)\right) .
$$

Thus, $D+\pi^{*}(A)$ is semi-ample at $x$.

As immediate consequences of Lemma 1.2, we have the following propositions. The first one is a characterization of weak positivity in terms of ample divisors.

Proposition $1.3(\operatorname{char}(k) \geq 0)$. Let $X$ be a quasi-projective variety over $k, x$ a point of $X$, and $D$ a $\mathbb{Q}$-Cartier divisor on $X$. Then, the following are equivalent.

(1) $D$ is weakly positive at $x$.

(2) For any ample $\mathbb{Q}$-Cartier divisors $A$ on $X, D+A$ is semi-ample at $x$.

(3) There is an ample $\mathbb{Q}$-Cartier divisor $A$ on $X$ such that $D+\epsilon A$ is semi-ample at $x$ for any positive rational numbers $\epsilon$.

Proposition $1.4(\operatorname{char}(k) \geq 0)$. Let $X$ be a normal quasi-projective variety over $k, X_{0}$ a Zariski open set of $X$, and $x$ a point of $X_{0}$. Let $D$ be $a \mathbb{Q}$-Cartier divisor on $X$ and $D_{0}=\left.D\right|_{X_{0}}$. If $\operatorname{codim}\left(X \backslash X_{0}\right) \geq 2$, then we have the following.

(1) $D$ is semi-ample at $x$ if and only if $D_{0}$ is semi-ample at $x$.

(2) $D$ is weakly positive at $x$ if and only if $D_{0}$ is weakly positive at $x$.

Next, let us consider functorial properties of semi-ampleness and weak positivity under pull-back and push-forward.

Proposition $1.5(\operatorname{char}(k) \geq 0)$. Let $\pi: X \rightarrow Y$ be a morphism of quasi-projective varieties over $k$. Let $D$ be a $\mathbb{Q}$-Cartier divisor on $Y$ and $x$ a point of $X$. If $\pi^{*}(D)$ is defined, then we have the following. (Note that even if $\pi^{*}(D)$ is not defined, there is a $\mathbb{Q}$-Cartier divisor $D^{\prime}$ such that $D^{\prime} \sim D$ and $\pi^{*}\left(D^{\prime}\right)$ is defined.)

(1) If $D$ is semi-ample at $\pi(x)$, then $\pi^{*}(D)$ is semi-ample at $x$.

(2) If $D$ is weakly positive at $\pi(x)$, then $\pi^{*}(D)$ is weakly positive at $x$.

Proof. (1) By our assumption, $H^{0}\left(Y, \mathcal{O}_{Y}(n D)\right) \otimes \mathcal{O}_{Y} \rightarrow \mathcal{O}_{Y}(n D)$ is surjective at $\pi(x)$ for a sufficiently large $n$. Thus, $H^{0}\left(Y, \mathcal{O}_{Y}(n D)\right) \otimes \mathcal{O}_{X} \rightarrow \mathcal{O}_{X}\left(n \pi^{*}(D)\right)$ is surjective at $x$. Here let us consider the following commutative diagram:

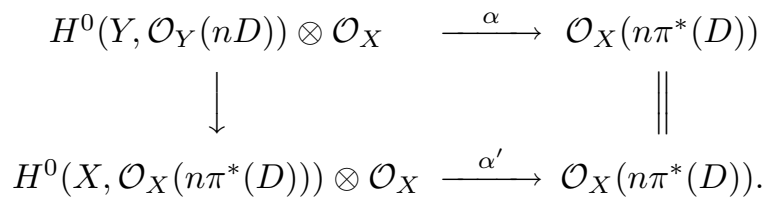

Since $\alpha$ is surjective at $x$, so is $\alpha^{\prime}$. Therefore, $\pi^{*}(D)$ is semi-ample at $x$. 
(2) Let $A$ be an ample divisor on $Y$ such that $\pi^{*}(A)$ is defined. Then, by Lemma $1.2, D+(1 / n) A$ is semi-ample at $\pi(x)$ for all $n>0$. Thus, by $(1), \pi^{*}(D)+$ $(1 / n) \pi^{*}(A)$ is semi-ample at $x$ for all $n>0$. Therefore, $\pi^{*}(D)$ is weakly positive at $x$.

Proposition $1.6(\operatorname{char}(k) \geq 0)$. Let $\pi: X \rightarrow Y$ be a surjective, proper and generically finite morphism of normal quasi-projective varieties over $k$. Let $D$ be a $\mathbb{Q}$ Cartier divisor on $X$ and $y$ a point of $Y$ such that $\pi_{*}(D)$ is a $\mathbb{Q}$-Cartier divisor on $Y$ and $\pi$ is finite over $y$. We set $\pi^{-1}(y)=\left\{x_{1}, \ldots, x_{n}\right\}$. Then, we have the following.

(1) If $D$ is semi-ample at $x_{1}, \ldots, x_{n}$, then $\pi_{*}(D)$ is semi-ample at $y$.

(2) If $D$ is weakly positive at $x_{1}, \ldots, x_{n}$, then $\pi_{*}(D)$ is weakly positive at $y$.

Proof. (1) Clearly, we may assume that $D$ is a Cartier divisor. If we take a sufficiently large integer $m$, then $H^{0}\left(X, \mathcal{O}_{X}(m D)\right) \otimes \mathcal{O}_{X} \rightarrow \mathcal{O}_{X}(m D)$ is surjective at $x_{1}, \ldots, x_{n}$. Thus, there are sections $s_{1}, \ldots, s_{n}$ of $H^{0}\left(X, \mathcal{O}_{X}(m D)\right)$ with $s_{i}\left(x_{i}\right) \neq 0$ for all $i=1, \ldots, n$. For $\alpha=\left(\alpha_{1}, \ldots, \alpha_{n}\right) \in k^{n}$, we set $s_{\alpha}=\alpha_{1} s_{1}+\cdots+\alpha_{n} s_{n}$. Further, we set $V_{i}=\left\{\alpha \in k^{n} \mid s_{\alpha}\left(x_{i}\right)=0\right\}$. Then, $\operatorname{dim} V_{i}=n-1$ for all $i$. Thus, since $\#(k)=\infty$, there is $\alpha \in k^{n}$ with $\alpha \notin V_{1} \cup \cdots \cup V_{r}$, i.e., $s_{\alpha}\left(x_{i}\right) \neq 0$ for all $i$. Let us consider a divisor $E=\operatorname{div}\left(s_{\alpha}\right)$. Then, $E \sim m D$. Thus, $\pi_{*}(E) \sim m \pi_{*}(D)$. Here, $x_{i} \notin E$ for all $i$. Hence, $y \notin \pi_{*}(E)$. Therefore, we get our assertion.

(2) Let $A$ be an ample divisor on $Y$. We set $D_{m}=D+(1 / m) \pi^{*}(A)$. Then, by Lemma $1.2, D_{m}$ is semi-ample at $x_{1}, \ldots, x_{n}$. Thus, by $(1), \pi_{*}\left(D_{m}\right)=\pi_{*}(D)+$ $(1 / m) \operatorname{deg}(\pi) A$ is semi-ample at $y$. Therefore, $\pi_{*}(D)$ is weakly positive at $y$.

Finally, let us consider semi-ampleness and weak positivity over an open set. Let $X$ be a quasi-projective variety over $k, U$ a Zariski open set of $X$, and $D$ a $\mathbb{Q}$-Cartier divisor on $X$. We say $D$ is semi-ample over $U$ (resp. weakly positive over $U$ ) if $D$ is semi-ample (resp. weakly positive) at all points of $U$. Then, we can easily see the following.

Proposition $1.7(\operatorname{char}(k) \geq 0)$. (1) If $D$ is semi-ample over $U$, then there is a positive integer $n$ such that $n D$ is a Cartier divisor and $H^{0}\left(X, \mathcal{O}_{X}(n D)\right) \otimes$ $\mathcal{O}_{X} \rightarrow \mathcal{O}_{X}(n D)$ is surjective on $U$.

(2) If $D$ is weakly positive over $U$, then, for any ample $\mathbb{Q}$-Cartier divisors $A$ on $X$, there is a positive integer $n$ such that $n(D+A)$ is a Cartier divisor and

$$
H^{0}\left(X, \mathcal{O}_{X}(n(D+A))\right) \otimes \mathcal{O}_{X} \rightarrow \mathcal{O}_{X}(n(D+A))
$$

is surjective on $U$.

\section{Proof of the Relative Bogomolov's inequality}

Let $X$ be an algebraic variety over $k, x$ a point of $X$, and $E$ a coherent $\mathcal{O}_{X^{-}}$ module on $X$. We say $E$ is generated by global sections at $x$ if $H^{0}(X, E) \otimes \mathcal{O}_{X} \rightarrow E$ is surjective at $x$. Let us begin with the following proposition.

Proposition $2.1(\operatorname{char}(k) \geq 0)$. Let $X$ be a smooth algebraic variety over $k, E$ a coherent $\mathcal{O}_{X}$-module, and $x$ a point of $X$. If $E$ is generated by global sections at $x$ and $E$ is free at $x$, then $\operatorname{det}(E)$ is generated by global sections at $x$, where $\operatorname{det}(E)$ is the determinant line bundle of $E$ in the sense of [10]. 
Proof. Let $T$ be the torsion part of $E$. Then, $\operatorname{det}(E)=\operatorname{det}(E / T) \otimes \operatorname{det}(T)$. If we set

$$
D=\sum_{\substack{P \in X, \operatorname{depth}(P)=1}} \operatorname{length}\left(T_{P}\right) \overline{\{P\}},
$$

then $\operatorname{det}(T) \simeq \mathcal{O}_{X}(D)$, where $\overline{\{P\}}$ is the Zariski closure of $\{P\}$ in $X$. Here since $E$ is free at $x, x \notin \operatorname{Supp}(D)$. Thus, $\operatorname{det}(T)$ is generated by global sections at $x$. Moreover, it is easy to see that $E / T$ is generated by global sections at $x$. Therefore, to prove our proposition, we may assume that $E$ is a torsion free sheaf.

Let $r$ be the rank of $E$ and $\kappa(x)$ the residue field of $x$. Then, by our assumption, there are sections $s_{1}, \ldots, s_{r}$ of $E$ such that $\left\{s_{i}(x)\right\}$ forms a basis of $E \otimes \kappa(x)$. Thus, $s=s_{1} \wedge \cdots \wedge s_{r}$ gives rise to a section of $\operatorname{det}(E)=\left(\wedge^{r} E\right)^{* *}$ with $s(y) \neq 0$. Hence, we get our proposition.

Next let us consider the following proposition.

Proposition $2.2(\operatorname{char}(k) \geq 0)$. Let $\pi: X \rightarrow Y$ be a proper and generically finite morphism of algebraic varieties over $k$. Let $y$ be a point of $Y$ such that $\pi$ is finite over $y$.

(1) Let $\phi: E \rightarrow Q$ be a homomorphism of coherent $\mathcal{O}_{X}$-modules. If $\phi$ is surjective at each point of $\pi^{-1}(y)$ and $\pi_{*}(E)$ is generated by global sections at $y$, then $\pi_{*}(Q)$ is generated by global sections at $y$.

(2) Let $E_{1}$ and $E_{2}$ be coherent $\mathcal{O}_{X}$-modules. If $\pi_{*}\left(E_{1}\right)$ and $\pi_{*}\left(E_{2}\right)$ are generated by global sections at $y$, then so is $\pi_{*}\left(E_{1} \otimes E_{2}\right)$ at $y$.

(3) Let $E$ be a coherent $\mathcal{O}_{X}$-module. If $\pi_{*}(E)$ is generated by global sections at $y$, then so is $\pi_{*}\left(\operatorname{Sym}^{n}(E)\right)$ at $y$ for every $n>0$.

Proof. (1) We can take an affine open neighborhood $U$ of $y$ such that $\pi$ is finite over $U$ and $\phi: E \rightarrow Q$ is surjective over $\pi^{-1}(U)$. Thus, $\pi_{*}(E) \rightarrow \pi_{*}(Q)$ is surjective at $y$. Hence, considering the following diagram:

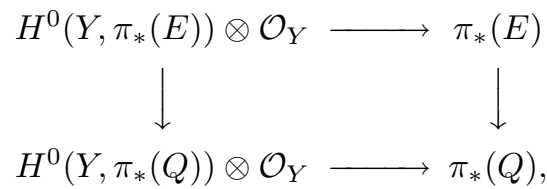

we have our assertion.

(2) Let $U$ be an affine open neighborhood of $y$ such that $\pi$ is finite over $U$. We set $U=\operatorname{Spec}(A)$ for some integral domain $A$. Since $\pi$ is finite over $U$, there is an integral domain $B$ with $\pi^{-1}(U)=\operatorname{Spec}(B)$. Here we take $B$-modules $M_{1}$ and $M_{2}$ such that $M_{1}$ and $M_{2}$ give rise to $\left.E_{1}\right|_{\pi^{-1}(U)}$ and $\left.E_{2}\right|_{\pi^{-1}(U)}$ respectively. Then, we have a natural surjective homomorphism $M_{1} \otimes_{A} M_{2} \rightarrow M_{1} \otimes_{B} M_{2}$. This shows us that $\pi_{*}(E) \otimes \pi_{*}\left(E_{2}\right) \rightarrow \pi_{*}\left(E_{1} \otimes E_{2}\right)$ is surjective at $y$. Here, let us consider the following diagram:

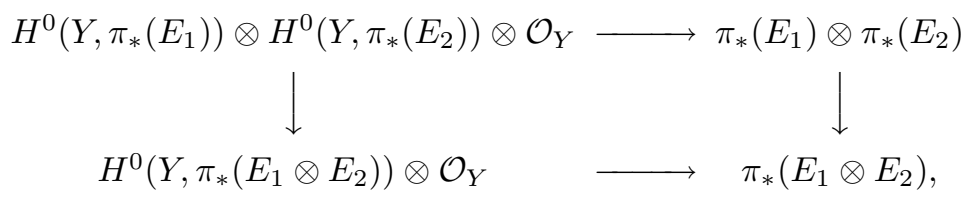

where $H^{0}\left(Y, \pi_{*}\left(E_{1}\right)\right) \otimes H^{0}\left(Y, \pi_{*}\left(E_{2}\right)\right) \otimes \mathcal{O}_{Y} \rightarrow \pi_{*}\left(E_{1}\right) \otimes \pi_{*}\left(E_{2}\right)$ is surjective at $y$ by our assumption. Thus, we get (2). 
(3) This is a consequence of (1) and (2) because we have a natural surjective homomorphism $E^{\otimes n} \rightarrow \operatorname{Sym}^{n}(E)$.

Before starting the main theorem, we need to prepare the following formula derived from the Grothendieck-Riemann-Roch theorem.

Lemma $2.3(\operatorname{char}(k) \geq 0)$. Let $X$ and $Y$ be algebraic varieties over $k$, and let $f: X \rightarrow Y$ be a surjective and projective morphism over $k$ of $\operatorname{dim} f=d$. Let $L$ and $A$ be line bundles on $X$. If $Y$ is smooth, then there are elements $Z_{1}, \ldots, Z_{d}$ of $A_{\operatorname{dim} Y-1}(Y) \otimes \mathbb{Q}$ such that

$$
c_{1}\left(R f_{*}\left(L^{\otimes n} \otimes A\right)\right) \cap[Y]=\frac{f_{*}\left(c_{1}(L)^{d+1} \cap[X]\right)}{(d+1) !} n^{d+1}+\sum_{i=0}^{d} Z_{i} n^{i}
$$

for all $n>0$.

Proof. We use the same symbols as in [5]. First of all, $R f_{*}\left(L^{\otimes n} \otimes A\right) \in K^{\circ}(Y)$ because $Y$ is smooth. Thus, by [5, Theorem 18.3, (1) and (2)], i.e., the RiemannRoch theorem for singular varieties,

$$
\operatorname{ch}\left(R f_{*}\left(L^{\otimes n} \otimes A\right)\right) \cap \tau_{Y}\left(\mathcal{O}_{Y}\right)=f_{*}\left(\operatorname{ch}\left(L^{\otimes n} \otimes A\right) \cap \tau_{X}\left(\mathcal{O}_{X}\right)\right) .
$$

Since $\tau_{X}\left(\mathcal{O}_{X}\right)=[X]+$ terms of dimension $<\operatorname{dim} X$ by $[5$, Theorem $18.3,(5)]$, it is easy to see that there are $T_{0}, \ldots, T_{d} \in A_{\operatorname{dim} Y-1}(X) \otimes \mathbb{Q}$ such that

$$
\left(\operatorname{ch}\left(L^{\otimes n} \otimes A\right) \cap \tau_{X}\left(\mathcal{O}_{X}\right)\right)_{\operatorname{dim} Y-1}=\frac{c_{1}(L)^{d+1} \cap[X]}{(d+1) !} n^{d+1}+\sum_{i=0}^{d} T_{i} n^{i} .
$$

Thus,

$$
f_{*}\left(\operatorname{ch}\left(L^{\otimes n} \otimes A\right) \cap \tau_{X}\left(\mathcal{O}_{X}\right)\right)_{\operatorname{dim} Y-1}=\frac{f_{*}\left(c_{1}(L)^{d+1} \cap[X]\right)}{(d+1) !} n^{d+1}+\sum_{i=0}^{d} f_{*}\left(T_{i}\right) n^{i} .
$$

On the other hand, since $\tau_{Y}\left(\mathcal{O}_{Y}\right)=[Y]+$ terms of $\operatorname{dimension}<\operatorname{dim} Y$, if we denote by $S$ the $(\operatorname{dim} Y-1)$-dimensional part of $\tau_{Y}\left(\mathcal{O}_{Y}\right)$, then

$$
\begin{aligned}
\left(\operatorname{ch}\left(R f_{*}\left(L^{\otimes n} \otimes A\right)\right)\right. & \left.\cap \tau_{Y}\left(\mathcal{O}_{Y}\right)\right)_{\operatorname{dim} Y-1} \\
& =c_{1}\left(R f_{*}\left(L^{\otimes n} \otimes A\right)\right) \cap[Y]+\operatorname{rk}\left(R f_{*}\left(L^{\otimes n} \otimes A\right)\right) S .
\end{aligned}
$$

Here, $\operatorname{rk}\left(R f_{*}\left(L^{\otimes n} \otimes A\right)\right)=\chi\left(X_{\eta},\left(L^{\otimes n} \otimes A\right)_{\eta}\right)$ is a polynomial of $n$ with degree $d$ at most, where $\eta$ is the generic point of $Y$. Thus, combining (2.3.1), (2.3.2) and (2.3.3), we have our lemma.

Let us start the main theorem of this paper.

Theorem $2.4(\operatorname{char}(k) \geq 0)$. Let $X$ be a quasi-projective variety over $k, Y a$ smooth quasi-projective variety over $k$, and $f: X \rightarrow Y$ a surjective and projective morphism over $k$ of $\operatorname{dim} f=1$. Let $F$ be a locally free sheaf on $X$ with $f_{*}\left(c_{1}(F) \cap[X]\right)=0$, and $y$ a point of $Y$. We assume that $f$ is flat over $y$, and that there are line bundles $L$ and $M$ on the geometric fiber $X_{\bar{y}}$ over $y$ such that

$$
H^{0}\left(X_{\bar{y}}, \operatorname{Sym}^{m}\left(F_{\bar{y}}\right) \otimes L\right)=H^{1}\left(X_{\bar{y}}, \operatorname{Sym}^{m}\left(F_{\bar{y}}\right) \otimes M\right)=0
$$

for $m \gg 0$. Then, $\left.f_{*}\left(\left(c_{2}(F)-c_{1}(F)^{2}\right) \cap[X]\right)\right)$ is weakly positive at $y$. 
Proof. Let $A$ be a very ample line bundle on $X$ such that $A_{\bar{y}} \otimes L$ and $A_{\bar{y}} \otimes M^{\otimes-1}$ are very ample on $X_{\bar{y}}$. First of all, we would like to see the following.

Claim 2.4.1. $H^{0}\left(X_{y}, \operatorname{Sym}^{m}\left(F_{y}\right) \otimes A_{y}^{\otimes-1}\right)=H^{1}\left(X_{y}, \operatorname{Sym}^{m}\left(F_{y}\right) \otimes A_{y}\right)=0$ for $m \gg$ 0 .

In general, for a coherent sheaf $G$ on $X_{y}, H^{i}\left(X_{\bar{y}}, G \otimes_{\kappa(y)} \overline{\kappa(y)}\right)=H^{i}\left(X_{y}, G\right) \otimes_{\kappa(y)}$ $\overline{\kappa(y)}$ for all $i \geq 0$. Thus, it is sufficient to show that

$$
H^{0}\left(X_{\bar{y}}, \operatorname{Sym}^{m}\left(F_{\bar{y}}\right) \otimes A_{\bar{y}}^{\otimes-1}\right)=H^{1}\left(X_{\bar{y}}, \operatorname{Sym}^{m}\left(F_{\bar{y}}\right) \otimes A_{\bar{y}}\right)=0
$$

for $m \gg 0$. Since $A_{\bar{y}} \otimes L$ is very ample and $\#(\overline{\kappa(y)})=\infty$, there is a section $s \in H^{0}\left(X_{\bar{y}}, A_{\bar{y}} \otimes L\right)$ such that $s \neq 0$ in $\left(A_{\bar{y}} \otimes L\right) \otimes \kappa(P)$ for any associated points $P$ of $X_{\bar{y}}$. Then, $\mathcal{O}_{X_{\bar{y}}} \stackrel{\times s}{\longrightarrow} A_{\bar{y}} \otimes L$ is injective. Thus, tensoring the above injection with $\operatorname{Sym}^{m}\left(F_{\bar{y}}\right) \otimes A_{\bar{y}}^{\otimes-1}$, we have an injection

$$
\operatorname{Sym}^{m}\left(F_{\bar{y}}\right) \otimes A_{\bar{y}}^{\otimes-1} \rightarrow \operatorname{Sym}^{m}\left(F_{\bar{y}}\right) \otimes L .
$$

Hence, $H^{0}\left(X_{\bar{y}}, \operatorname{Sym}^{m}\left(F_{\bar{y}}\right) \otimes A_{\bar{y}}^{\otimes-1}\right)=0$ for $m \gg 0$.

In the same way, there is a section $s^{\prime} \in H^{0}\left(X_{\bar{y}}, A_{\bar{y}} \otimes M^{\otimes-1}\right)$ such that $s^{\prime} \neq 0$ in $\left(A_{\bar{y}} \otimes M^{\otimes-1}\right) \otimes \kappa(P)$ for any associated points $P$ of $X_{\bar{y}}$. Then, $\mathcal{O}_{X_{\bar{y}}} \stackrel{\times s^{\prime}}{\longrightarrow} A_{\bar{y}} \otimes M^{\otimes-1}$ is injective and its cokernel $T$ has the 0-dimensional support. Thus, tensoring an exact sequence

$$
0 \rightarrow \mathcal{O}_{X_{\bar{y}}} \rightarrow A_{\bar{y}} \otimes M^{\otimes-1} \rightarrow T \rightarrow 0
$$

with $\operatorname{Sym}^{m}\left(F_{\bar{y}}\right) \otimes M$, we obtain an exact sequence

$$
0 \rightarrow \operatorname{Sym}^{m}\left(F_{\bar{y}}\right) \otimes M \rightarrow \operatorname{Sym}^{m}\left(F_{\bar{y}}\right) \otimes A_{\bar{y}} \rightarrow \operatorname{Sym}^{m}\left(F_{\bar{y}}\right) \otimes M \otimes T \rightarrow 0 .
$$

Hence, we get a surjection

$$
H^{1}\left(X_{\bar{y}}, \operatorname{Sym}^{m}\left(F_{\bar{y}}\right) \otimes M\right) \rightarrow H^{1}\left(X_{\bar{y}}, \operatorname{Sym}^{m}\left(F_{\bar{y}}\right) \otimes A_{\bar{y}}\right) .
$$

Therefore, $H^{1}\left(X_{\bar{y}}, \operatorname{Sym}^{m}\left(F_{\bar{y}}\right) \otimes A_{\bar{y}}\right)=0$ for $m \gg 0$.

Since $X$ is an integral scheme over $k$ of dimension greater than or equal to 2 , and $X_{y}$ is a 1-dimensional scheme over $\kappa(y)$, by virtue of [9, Theorem 6.10], there is $B \in\left|A^{\otimes 2}\right|$ such that $B$ is integral, and that $B \cap X_{y}$ is finite, i.e., $B$ is finite over $y$. Let $\pi: B \rightarrow Y$ be the morphism induced by $f$. Let $H$ be an ample line bundle on $Y$ such that $\pi_{*}\left(F_{B}\right) \otimes H$ and $\pi_{*}\left(A_{B}\right) \otimes H$ are generated by global sections at $y$, where $F_{B}=\left.F\right|_{B}$ and $A_{B}=\left.A\right|_{B}$.

Let $\mu: P=\operatorname{Proj}\left(\bigoplus_{m=0}^{\infty} \operatorname{Sym}^{m}(F)\right) \rightarrow X$ be the projective bundle and $\mathcal{O}_{P}(1)$ the tautological line bundle on $P$. We set $h=f \cdot \mu: P \rightarrow Y$. Let us consider

$$
c_{1}\left(R h_{*}\left(\left(\mathcal{O}_{P}(1) \otimes h^{*}(H)\right)^{\otimes m} \otimes \mu^{*}\left(A^{\otimes-1}\right) \otimes h^{*}(H)\right)\right) \cap[Y]
$$

for $m \gg 0$. By Lemma 2.3, there are elements $Z_{0}, \ldots, Z_{r}$ of $A_{\operatorname{dim} Y-1}(Y) \otimes \mathbb{Q}$ such that

$$
\begin{aligned}
c_{1}\left(R h_{*}\left(\left(\mathcal{O}_{P}(1) \otimes h^{*}(H)\right)^{\otimes m} \otimes \mu^{*}\left(A^{\otimes-1}\right) \otimes h^{*}(H)\right)\right) \cap[Y] \\
\quad=\frac{h_{*}\left(c_{1}\left(\mathcal{O}_{P}(1) \otimes h^{*}(H)\right)^{r+1} \cap[P]\right)}{(r+1) !} m^{r+1}+\sum_{i=0}^{r} Z_{i} m^{i},
\end{aligned}
$$


where $r$ is the rank of $F$. Here

$$
\left\{\begin{array}{l}
\mu_{*}\left(c_{1}\left(\mathcal{O}_{P}(1)\right)^{r+1} \cap[P]\right)=\left(c_{1}(F)^{2}-c_{2}(F)\right) \cap[X], \\
\mu_{*}\left(c_{1}\left(\mathcal{O}_{P}(1)\right)^{r} \cap[P]\right)=c_{1}(F) \cap[X], \\
\mu_{*}\left(c_{1}\left(\mathcal{O}_{P}(1)\right)^{r-1} \cap[P]\right)=[X], \\
\mu_{*}\left(c_{1}\left(\mathcal{O}_{P}(1)\right)^{j} \cap[P]\right)=0 \quad(0 \leq j<r-1) .
\end{array}\right.
$$

Thus, by using the projection formula, we have

$$
\begin{aligned}
& h_{*}\left(c_{1}\left(\mathcal{O}_{P}(1) \otimes h^{*}(H)\right)^{r+1} \cap[P]\right) \\
&=f_{*} \mu_{*}\left(\sum_{i=0}^{r+1} \mu^{*} f^{*}\left(c_{1}(H)^{i}\right) \cap\left(c_{1}\left(\mathcal{O}_{P}(1)\right)^{r+1-i} \cap[P]\right)\right) \\
&=f_{*}\left(\left(c_{1}(F)^{2}-c_{2}(F)\right) \cap[X]\right) \\
&+r f_{*}\left(f^{*}\left(c_{1}(H)\right) \cap\left(c_{1}(F) \cap[X]\right)\right) \\
& \quad+\frac{r(r+1)}{2} f_{*}\left(f^{*}\left(c_{1}(H)^{2}\right) \cap[X]\right) \\
&=-f_{*}\left(\left(c_{2}(F)-c_{1}(F)^{2}\right) \cap[X]\right)
\end{aligned}
$$

because $f_{*}\left(c_{1}(F) \cap[X]\right)=0$ and $f_{*}([X])=0$. Moreover,

$$
\begin{aligned}
R \mu_{*}\left(\left(\mathcal{O}_{P}(1) \otimes h^{*}(H)\right)^{\otimes m} \otimes \mu^{*}\left(A^{\otimes-1}\right)\right. & \left.\otimes h^{*}(H)\right) \\
& =\operatorname{Sym}^{m}\left(F \otimes f^{*}(H)\right) \otimes A^{\otimes-1} \otimes f^{*}(H) .
\end{aligned}
$$

Therefore, we get

$$
\begin{aligned}
\sum_{i \geq 0}(-1)^{i} c_{1}\left(R^{i} f_{*}\left(\operatorname{Sym}^{m}\left(F \otimes f^{*}(H)\right) \otimes A^{\otimes-1} \otimes f^{*}(H)\right)\right) \cap[Y] \\
=-\frac{1}{(r+1) !} f_{*}\left(\left(c_{2}(F)-c_{1}(F)^{2}\right) \cap[X]\right) m^{r+1}+\sum_{i=0}^{r} Z_{i} m^{i} .
\end{aligned}
$$

Here we claim the following.

Claim 2.4.2. If $m \gg 0$, then we have the following.

(a) $c_{1}\left(R^{i} f_{*}\left(\operatorname{Sym}^{m}\left(F \otimes f^{*}(H)\right) \otimes A^{\otimes-1} \otimes f^{*}(H)\right)\right) \cap[Y]=0$ for all $i \geq 2$.

(b) $f_{*}\left(\operatorname{Sym}^{m}\left(F \otimes f^{*}(H)\right) \otimes A^{\otimes-1} \otimes f^{*}(H)\right)=0$.

(c) $R^{1} f_{*}\left(\operatorname{Sym}^{m}\left(F \otimes f^{*}(H)\right) \otimes A^{\otimes-1} \otimes f^{*}(H)\right)$ is free at $y$.

(d) $R^{1} f_{*}\left(\operatorname{Sym}^{m}\left(F \otimes f^{*}(H)\right) \otimes A \otimes f^{*}(H)\right)=0$ around $y$.

(a): Let $Y^{\prime}$ be the maximal open set of $Y$ such that $f$ is flat over $Y^{\prime}$. If $i \geq 2$, then the support of $R^{i} f_{*}\left(\operatorname{Sym}^{m}\left(F \otimes f^{*}(H)\right) \otimes A^{\otimes-1} \otimes f^{*}(H)\right)$ is contained in $Y \backslash Y^{\prime}$. Here $\operatorname{codim}\left(Y \backslash Y^{\prime}\right) \geq 2$. Thus, we get (a).

(b) and (c): By Claim 2.4.1, $H^{0}\left(X_{y}, \operatorname{Sym}^{m}\left(F_{y}\right) \otimes A_{y}^{\otimes-1}\right)=0$ for $m \gg 0$. Thus, using the upper-semicontinuity of dimension of cohomology groups, there is an open neighborhood $U_{m}$ of $y$ such that $f$ is flat over $U_{m}$ and

$$
H^{0}\left(X_{y^{\prime}}, \operatorname{Sym}^{m}\left(F_{y^{\prime}}\right) \otimes A_{y^{\prime}}^{\otimes-1}\right)=0
$$

for all $y^{\prime} \in U_{m}$, which implies (b) because $f_{*}\left(\operatorname{Sym}^{m}\left(F \otimes f^{*}(H)\right) \otimes A^{\otimes-1} \otimes f^{*}(H)\right)$ is torsion free. Here, since $f$ is flat over $U_{m}, \chi\left(X_{y^{\prime}}, \operatorname{Sym}^{m}\left(F_{y^{\prime}}\right) \otimes A_{y^{\prime}}^{\otimes-1}\right)$ is a constant with respect to $y^{\prime} \in U_{m}$. Therefore, so is $h^{1}\left(X_{y^{\prime}}, \operatorname{Sym}^{m}\left(F_{y^{\prime}}\right) \otimes A_{y^{\prime}}^{\otimes-1}\right)$ with respect to $y^{\prime} \in U_{m}$. Thus, we have (c). 
(d): By virtue of Claim 2.4.1, $H^{1}\left(X_{y}, \operatorname{Sym}^{m}\left(F_{y}\right) \otimes A_{y}\right)=0$ for $m \gg 0$. Thus, there is an open neighborhood $U_{m}^{\prime}$ of $y$ such that $f$ is flat over $U_{m}^{\prime}$ and

$$
H^{1}\left(X_{y^{\prime}}, \operatorname{Sym}^{m}\left(F_{y^{\prime}}\right) \otimes A_{y^{\prime}}\right)=0
$$

for all $y^{\prime} \in U_{m}^{\prime}$. Hence, we can see (d).

By (a) and (b) of Claim 2.4.2,

$$
\begin{aligned}
& \frac{1}{(r+1) !} f_{*}\left(\left(c_{2}(F)-c_{1}(F)^{2}\right) \cap[X]\right) \\
& \quad=\frac{c_{1}\left(R^{1} f_{*}\left(\operatorname{Sym}^{m}\left(F \otimes f^{*}(H)\right) \otimes A^{\otimes-1} \otimes f^{*}(H)\right)\right) \cap[Y]}{m^{r+1}}+\sum_{i=0}^{r} \frac{Z_{i}}{m^{r+1-i}} .
\end{aligned}
$$

Hence, it is sufficient to show that

$$
c_{1}\left(R^{1} f_{*}\left(\operatorname{Sym}^{m}\left(F \otimes f^{*}(H)\right) \otimes A^{\otimes-1} \otimes f^{*}(H)\right)\right) \cap[Y]
$$

is semi-ample at $y$.

Since $\pi_{*}\left(F_{B} \otimes \pi^{*}(H)\right)$ and $\pi_{*}\left(A_{B} \otimes \pi^{*}(H)\right)$ are generated by global sections at $y$, by (2) and (3) of Proposition 2.2, $\pi_{*}\left(\operatorname{Sym}^{m}\left(F_{B} \otimes \pi^{*}(H)\right) \otimes A_{B} \otimes \pi^{*}(H)\right)$ is generated by global sections at $y$. On the other hand, a short exact sequence

$$
\begin{aligned}
0 \rightarrow \operatorname{Sym}^{m}\left(F \otimes f^{*}(H)\right) \otimes A^{\otimes-1} \otimes & f^{*}(H) \rightarrow \operatorname{Sym}^{m}\left(F \otimes f^{*}(H)\right) \otimes A \otimes f^{*}(H) \\
& \rightarrow \operatorname{Sym}^{m}\left(F_{B} \otimes \pi^{*}(H)\right) \otimes A_{B} \otimes \pi^{*}(H) \rightarrow 0
\end{aligned}
$$

gives rise to an exact sequence

$$
\begin{aligned}
& 0 \rightarrow f_{*}\left(\operatorname{Sym}^{m}\left(F \otimes f^{*}(H)\right) \otimes A \otimes f^{*}(H)\right) \rightarrow \pi_{*}\left(\operatorname{Sym}^{m}\left(F_{B} \otimes \pi^{*}(H)\right) \otimes A_{B} \otimes \pi^{*}(H)\right) \\
\rightarrow & R^{1} f_{*}\left(\operatorname{Sym}^{m}\left(F \otimes f^{*}(H)\right) \otimes A^{\otimes-1} \otimes f^{*}(H)\right) \rightarrow R^{1} f_{*}\left(\operatorname{Sym}^{m}\left(F \otimes f^{*}(H)\right) \otimes A \otimes f^{*}(H)\right) .
\end{aligned}
$$

Thus, by (d) of Claim 2.4.2,

$$
\begin{aligned}
\phi_{m}: \pi_{*}\left(\operatorname{Sym}^{m}\left(F_{B} \otimes \pi^{*}(H)\right) \otimes\right. & \left.A_{B} \otimes \pi^{*}(H)\right) \\
& \rightarrow R^{1} f_{*}\left(\operatorname{Sym}^{m}\left(F \otimes f^{*}(H)\right) \otimes A^{\otimes-1} \otimes f^{*}(H)\right)
\end{aligned}
$$

is surjective at $y$. Therefore, applying (1) of Proposition 2.2 to the case where $\operatorname{id}_{Y}: Y \rightarrow Y$ and $\phi=\phi_{m}$, we have that $R^{1} f_{*}\left(\operatorname{Sym}^{m}\left(F \otimes f^{*}(H)\right) \otimes A^{\otimes-1} \otimes f^{*}(H)\right)$ is generated by global sections at $y$. Moreover, by virtue of (c) of Claim 2.4.2, $R^{1} f_{*}\left(\operatorname{Sym}^{m}\left(F \otimes f^{*}(H)\right) \otimes A^{\otimes-1} \otimes f^{*}(H)\right)$ is free at $y$. Hence, by Proposition 2.1, $c_{1}\left(R^{1} f_{*}\left(\operatorname{Sym}^{m}\left(F \otimes f^{*}(H)\right) \otimes A^{\otimes-1} \otimes f^{*}(H)\right)\right) \cap[Y]$ is semi-ample at $y$.

As a corollary of Theorem 2.4, we have the following.

Corollary $2.5(\operatorname{char}(k)=0)$. Let $X$ be a quasi-projective variety over $k, Y a$ smooth quasi-projective variety over $k$, and $f: X \rightarrow Y$ a surjective and projective morphism over $k$ with $\operatorname{dim} f=1$. Let $E$ be a locally free sheaf on $X$ and $y$ a point of $Y$. If $f$ is flat over $y$, the geometric fiber $X_{\bar{y}}$ over $y$ is reduced and Gorenstein, and $E$ is semistable on each connected component of the normalization of $X_{\bar{y}}$, then $\operatorname{dis}_{X / Y}(E)$ is weakly positive at $y$.

Proof. We set $F=\mathcal{E}$ nd $(E)$. First, we claim the following.

Claim 2.5.1. $H^{0}\left(X_{\bar{y}}, \operatorname{Sym}^{m}\left(F_{\bar{y}}\right) \otimes A^{\otimes-1}\right)=0$ for any ample line bundles $A$ on $X_{\bar{y}}$ and any $m \geq 0$. 
Let $\pi: Z \rightarrow X_{\bar{y}}$ be the normalization of $X_{\bar{y}}$. The semistability of tensor products of semistable vector bundles in characteristic zero was studied by many authors [7], [8], [12], [11], etc. (You can find a new elementary algebraic proof in $\S 7$, which works in any characteristic under strong semistability.) Thus, by virtue of our assumption, $\operatorname{Sym}^{m}\left(\pi^{*}\left(F_{\bar{y}}\right)\right)$ is semistable and of degree 0 on each connected component of $Z$. Hence,

$$
H^{0}\left(Z, \pi^{*}\left(\operatorname{Sym}^{m}\left(F_{\bar{y}}\right) \otimes A^{\otimes-1}\right)\right)=0 .
$$

Here, since $\mathcal{O}_{X_{\bar{y}}} \rightarrow \pi_{*}\left(\mathcal{O}_{Z}\right)$ is injective, the above implies our claim.

Let $L$ be an ample line bundle on $X_{\bar{y}}$ such that $L \otimes \omega_{X_{\bar{y}}}^{\otimes-1}$ is ample. Here, since $F_{\bar{y}}^{*}=F_{\bar{y}}$, by using Serre's duality theorem, $H^{1}\left(X_{\bar{y}}, \operatorname{Sym}^{m}\left(F_{\bar{y}}\right) \otimes L\right)$ is isomorphic to the dual space of $H^{0}\left(X_{\bar{y}}, \operatorname{Sym}^{m}\left(F_{\bar{y}}\right) \otimes\left(L \otimes \omega_{X_{\bar{y}}}^{\otimes-1}\right)^{\otimes-1}\right)$. Thus, by Claim 2.5.1,

$$
H^{0}\left(X_{\bar{y}}, \operatorname{Sym}^{m}\left(F_{\bar{y}}\right) \otimes L^{\otimes-1}\right)=H^{1}\left(X_{\bar{y}}, \operatorname{Sym}^{m}\left(F_{\bar{y}}\right) \otimes L\right)=0
$$

for all $m \geq 0$. Hence, Theorem 2.4 implies our corollary because $c_{1}(F)=0$ and $c_{2}(F)=2 \operatorname{rk}(E) c_{2}(E)-(\operatorname{rk}(E)-1) c_{1}(E)^{2}$.

Remark 2.6. Even if $\operatorname{rk}(F)=1$, Theorem 2.4 is a non-trivial fact. For, if $f: X \rightarrow Y$ is a smooth surface fibred over a projective curve, then the assertion of it is nothing more than the Hodge index theorem.

\section{A WEAKLy POSITIVE Divisor on THE MODUli SPACE OF STABle CURVES}

Throughout this section, we assume that $\operatorname{char}(k)=0$.

Fix an integer $g \geq 2$ and a polynomial $P_{g}(n)=(6 n-1)(g-1)$. Let $H_{g} \subset$ Hilb $_{\mathbb{P}^{5 g-6}}^{P_{g}}$ be a subscheme of all tri-canonically embedded stable curves over $k$, $Z_{g} \subset H_{g} \times \mathbb{P}^{5 g-6}$ the universal tri-canonically embedded stable curves over $k$, and $\pi: Z_{g} \rightarrow H_{g}$ the natural projection. Let $\Delta$ be the minimal closed subset of $H_{g}$ such that $\pi$ is not smooth over a point of $\Delta$. Then, by [4, Theorem (1.6) and Corollary (1.9)], $Z_{g}$ and $H_{g}$ are quasi-projective and smooth over $k$, and $\Delta$ is a divisor with only normal crossings. Let $\Delta=\Delta_{0} \cup \cdots \cup \Delta_{[g / 2]}$ be the irreducible decomposition of $\Delta$ such that, if $x \in \Delta_{i} \backslash \operatorname{Sing}(\Delta)$, then $\pi^{-1}(x)$ is a stable curve with one node of type $i$. We set $U=H_{g} \backslash \Delta, H_{g}^{0}=H_{g} \backslash \operatorname{Sing}\left(\Delta_{1}+\cdots+\Delta_{[g / 2]}\right)$ and $Z_{g}^{0}=\pi^{-1}\left(H_{g}^{0}\right)$. In $[16, \S 3]$, we constructed a reflexive sheaf $F$ on $Z_{g}$ with the following properties.

(1) $F$ is locally free on $Z_{g}^{0}$.

(2) For each $y \in H_{g} \backslash\left(\Delta_{1} \cup \cdots \cup \Delta_{[g / 2]}\right)$,

$$
\left.F\right|_{\pi^{-1}(y)}=\operatorname{Ker}\left(H^{0}\left(\omega_{\pi^{-1}(y)}\right) \otimes \mathcal{O}_{\pi^{-1}(y)} \rightarrow \omega_{\pi^{-1}(y)}\right) .
$$

(3) $\operatorname{dis}_{Z_{g} / H_{g}}(F)=(8 g+4) \operatorname{det}\left(\pi_{*}\left(\omega_{Z_{g} / H_{g}}\right)\right)-g \Delta_{0}-\sum_{i=1}^{\left[\frac{g}{2}\right]} 4 i(g-i) \Delta_{i}$.

Actually, $F$ can be constructed as follows. First of all, we set

$$
E=\operatorname{Ker}\left(\pi^{*}\left(\pi_{*}\left(\omega_{Z_{g} / H_{g}}\right)\right) \rightarrow \omega_{Z_{g} / H_{g}}\right) .
$$

We would like to modify $E$ along singular fibers so that we can get our desired $F$. For this purpose, we consider $E^{0}=\left.E\right|_{Z_{g}^{0}}$. It is easy to see that $E^{0}$ is a locally free sheaf on $Z_{g}^{0}$. For each $i \geq 0$, we denote $\Delta_{i} \cap H_{g}^{0}$ by $\Delta_{i}^{0}$. If $i \geq 1$, then there is the irreducible decomposition $\pi^{-1}\left(\Delta_{i}^{0}\right)=C_{i}^{1} \cup C_{i}^{2}$ such that the generic fiber of 

$\underset{\text { set }}{\left.\pi\right|_{C_{i}^{1}}: C_{i}^{1} \rightarrow \Delta_{i}^{0}\left(\text { resp. }\left.\pi\right|_{C_{i}^{2}}: C_{i}^{2} \rightarrow \Delta_{i}^{0}\right) \text { is of genus } i(\text { resp. } g-i \text { ). Moreover, if we }}$

$$
Q_{i}^{j}=\operatorname{Ker}\left(\left(\left.\pi\right|_{C_{i}^{j}}\right)^{*}\left(\left.\pi\right|_{C_{i}^{j}}\right)_{*}\left(\omega_{C_{i}^{j} / \Delta_{i}^{0}}\right) \longrightarrow \omega_{C_{i}^{j} / \Delta_{i}^{0}}\right)
$$

for each $i \geq 1$ and $j=1,2$, then there is a natural surjective homomorphism

$$
\alpha_{i}^{j}:\left.E^{0}\right|_{C_{i}^{j}} \rightarrow Q_{i}^{j} .
$$

Here let us consider

$$
F^{0}=\operatorname{Ker}\left(\bigoplus_{i=1}^{\left[\frac{g}{2}\right]}\left(\alpha_{i}^{1} \oplus \alpha_{i}^{2}\right): E^{0} \longrightarrow \bigoplus_{i=1}^{\left[\frac{g}{2}\right]}\left(Q_{i}^{1} \oplus Q_{i}^{2}\right)\right) .
$$

As we showed in $[16, \S 3], F^{0}$ is a locally free sheaf on $Z_{g}^{0}$ with

$$
\operatorname{dis}_{Z_{g}^{0} / H_{g}^{0}}\left(F^{0}\right)=(8 g+4) \operatorname{det}\left(\pi_{*}\left(\omega_{Z_{g}^{0} / H_{g}^{0}}\right)\right)-g \Delta_{0}^{0}-\sum_{i=1}^{\left[\frac{g}{2}\right]} 4 i(g-i) \Delta_{i}^{0} .
$$

Let $\nu: Z_{g}^{0} \rightarrow Z_{g}$ be the natural inclusion map. Then $F$ can be defined by $\nu_{*}\left(F^{0}\right)$. In order to see (2), note that $E=F$ over $H_{g} \backslash\left(\Delta_{1} \cup \cdots \cup \Delta_{[g / 2]}\right)$ and $\pi^{*}\left(\pi_{*}\left(\omega_{Z_{g} / H_{g}}\right)\right) \rightarrow$ $\omega_{Z_{g} / H_{g}}$ is surjective on $H_{g} \backslash\left(\Delta_{1} \cup \cdots \cup \Delta_{[g / 2]}\right)$ (cf. [16, Proposition 2.1.3]).

Let $\overline{\mathcal{M}}_{g}$ (resp. $\mathcal{M}_{g}$ ) be the moduli space of stable (resp. smooth) curves of genus $g$ over $k$. Let $\phi: H_{g} \rightarrow \overline{\mathcal{M}}_{g}$ be the canonical morphism. Let $\lambda, \delta_{0}, \ldots, \delta_{[g / 2]} \in$ $\operatorname{Pic}\left(\overline{\mathcal{M}}_{g}\right) \otimes \mathbb{Q}$ such that $\phi^{*}(\lambda)=\operatorname{det}\left(\pi_{*}\left(\omega_{Z_{g} / H_{g}}\right)\right)$ and $\phi^{*}\left(\delta_{i}\right)=\Delta_{i}$ for all $0 \leq i \leq$ $[g / 2]$. Let us begin with the following lemma.

Lemma 3.1. Let $D$ be $a \mathbb{Q}$-Cartier divisor on $\overline{\mathcal{M}}_{g}$ and $x$ a point of $\overline{\mathcal{M}}_{g}$. If $\phi^{*}(D)$ is weakly positive at any points of $\phi^{-1}(x)$, then $D$ is weakly positive at $x$.

Proof. It is well known that there are a surjective finite morphism $\pi: Y \rightarrow \overline{\mathcal{M}}_{g}$ of normal projective varieties and a stable curve $f: X \rightarrow Y$ of genus $g$ such that the induced morphism $Y \rightarrow \overline{\mathcal{M}}_{g}$ by $f: X \rightarrow Y$ is $\pi$. Since $\pi_{*}\left(\pi^{*}(D)\right)=\operatorname{deg}(\pi) D$, by Proposition 1.6, it is sufficient to show that $\pi^{*}(D)$ is weakly positive at any points of $\pi^{-1}(x)$.

Let $y$ be a point of $\pi^{-1}(x)$. Then, there is a Zariski open neighborhood $U$ of $y$ such that $\left.f_{*}\left(\omega_{X / Y}^{\otimes 3}\right)\right|_{U}$ is free. Thus,

$$
\operatorname{Proj}\left(\bigoplus_{n=0}^{\infty} \operatorname{Sym}^{n}\left(\left.f_{*}\left(\omega_{X / Y}^{\otimes 3}\right)\right|_{U}\right)\right) \simeq U \times \mathbb{P}^{5 g-6}
$$

Therefore, there is a morphism $\mu: U \rightarrow H_{g}$ with $\left.\pi\right|_{U}=\phi \cdot \mu$. By abuse of notation, the induced rational map $Y \rightarrow H_{g}$ is denoted by $\mu$. Let $\nu: Y^{\prime} \rightarrow Y$ be a proper birational morphism of normal projective varieties such that $\mu^{\prime}=\mu \cdot \nu: Y^{\prime} \rightarrow H_{g}$ is a morphism and $\nu$ is an isomorphism over $\nu^{-1}(U)$. Then, we have the following diagram:

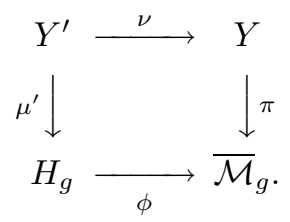


This diagram is commutative because $\phi \cdot \mu^{\prime}=\pi \cdot \nu$ over $\nu^{-1}(U)$. Hence, $\nu^{*}\left(\pi^{*}(D)\right)=$ $\mu^{\prime *}\left(\phi^{*}(D)\right)$. Moreover, $\nu_{*}\left(\nu^{*}\left(\pi^{*}(D)\right)\right)=\pi^{*}(D)$. Thus, by virtue of Proposition 1.6, in order to see that $\pi^{*}(D)$ is weakly positive at $y$, it is sufficient to check that $\mu^{\prime *}\left(\phi^{*}(D)\right)$ is weakly positive at $y \in \nu^{-1}(U)$.

By our assumption, $\phi^{*}(D)$ is weakly positive at $\mu^{\prime}(y)$ because $\phi\left(\mu^{\prime}(y)\right)=x$. Hence, by Proposition $1.5, \mu^{\prime *}\left(\phi^{*}(D)\right)$ is weakly positive at $y$.

Theorem 3.2. $(8 g+4) \lambda-g \delta_{0}-\sum_{i=1}^{[g / 2]} 4 i(g-i) \delta_{i}$ is weakly positive over $\mathcal{M}_{g}$. In particular, it is pseudo-effective, and numerically effective over $\mathcal{M}_{g}$.

Proof. Let $y$ be a point of $U=H_{g} \backslash \Delta$. By virtue of [18], $\left.F\right|_{\pi^{-1}(y)}$ is semistable. Thus, by Corollary 2.5, $\operatorname{dis}_{Z_{g}^{0}} / H_{g}^{0}\left(F^{0}\right)$ is weakly positive at $y$. Hence, by Proposition 1.4, so is $\operatorname{dis}_{Z_{g} / H_{g}}(F)$ at $y$ because $\operatorname{codim}\left(H_{g} \backslash H_{g}^{0}\right)=2$. Thus, $\operatorname{dis}_{Z_{g} / H_{g}}(F)$ is weakly positive over $U=\phi^{-1}\left(\mathcal{M}_{g}\right)$. Therefore, by virtue of Lemma 3.1, we get our theorem.

As a corollary, we have the following.

Corollary 3.3. Let $X$ be a smooth projective surface over $k, Y$ a smooth projective curve over $k$, and $f: X \rightarrow Y$ a semistable curve of genus $g \geq 2$ over $Y$. Then, we have the inequality

$$
(8 g+4) \operatorname{deg}\left(f_{*}\left(\omega_{X / Y}\right)\right) \geq g \delta_{0}(X / Y)+\sum_{i=1}^{[g / 2]} 4 i(g-i) \delta_{i}(X / Y),
$$

where $\delta_{i}(X / Y)$ is the number of nodes of type $i$ in all singular fibers of $f$.

Remark 3.4. We don't know the proof of Corollary 3.3 without using the moduli space $\overline{\mathcal{M}}_{g}$. Let $\mu: Y \rightarrow \overline{\mathcal{M}}_{g}$ be the morphism induced by $f: X \rightarrow Y$. Then, $\mu(Y)$ might pass through $\overline{\mathcal{M}}_{g} \backslash \phi\left(H_{g}^{0}\right)$. In this case, analyses of singular fibers only in $X$ seem to be very complicated.

\section{Cones of positive Divisors on the moduli Space of Stable CuRves}

Throughout this section, we assume that $\operatorname{char}(k)=0$.

Let $X$ be a projective variety over $k$ and $\mathcal{C}$ a certain family of complete irreducible curves on $X$. A $\mathbb{Q}$-Cartier divisor $D$ on $X$ is said to be numerically effective for $\mathcal{C}$ if $(D \cdot C) \geq 0$ for all $C \in \mathcal{C}$. We set

$$
\operatorname{Nef}(X, \mathcal{C})=\{D \in N S(X) \otimes \mathbb{Q} \mid D \text { is numerically effective for } \mathcal{C}\} .
$$

Moreover, for subsets $A$ and $B$ in $X$, we denote by $\operatorname{Cur}_{B}^{A}$ the set of all irreducible complete curves $C$ on $X$ with $C \subseteq A$ and $C \cap B \neq \emptyset$.

Let $g$ be an integer greater than or equal to $2, \mathcal{I}_{g}$ the locus of hyperelliptic curves in $\mathcal{M}_{g}, \overline{\mathcal{I}}_{g}$ the closure in $\overline{\mathcal{M}}_{g}$, and $\overline{\mathcal{M}}_{g}^{\text {one }}$ the set of all stable curves with at most one node, i.e., if we use the notation in the previous section,

$$
\overline{\mathcal{M}}_{g}^{\text {one }}=\phi\left(H_{g} \backslash \operatorname{Sing}\left(\Delta_{0}+\cdots+\Delta_{[g / 2]}\right)\right) .
$$

Let us begin with the following lemma.

Lemma 4.1. There are complete irreducible curves $C, C_{0}, \ldots, C_{[g / 2]}$ on $\overline{\mathcal{M}}_{g}$ with the following properties.

(1) $C, C_{0}, \ldots, C_{[g / 2]} \in \mathrm{Cur}_{\mathcal{I}_{g}}^{\overline{\mathcal{M}}_{g}^{\text {one }}}$. 
(2) $C \subset \mathcal{M}_{g}$.

(3) $C_{i} \subset \overline{\mathcal{I}}_{q}$ for all $0 \leq i \leq[g / 2]$.

(4) For all $0 \leq i, j \leq[\mathrm{g} / 2],\left(\delta_{i} \cdot C_{j}\right)$ is positive if $i=j$, and $\left(\delta_{i} \cdot C_{j}\right)=0$ if $i \neq j$.

Proof. Let $\overline{\mathcal{M}}_{g}^{s}$ be Satake's compactification of $\mathcal{M}_{g}$. Then, $\overline{\mathcal{M}}_{g}^{s}$ is projective and $\operatorname{codim}\left(\overline{\mathcal{M}}_{g}^{s} \backslash \mathcal{M}_{g}\right) \geq 2$. Pick up one point $P \in \mathcal{I}_{g}$. If we take general hyperplane sections $H_{1}, \ldots, H_{3 g-4}$ passing through $P$, then $C=H_{1} \cap \ldots \cap H_{3 g-4}$ is a complete irreducible curve with $C \subseteq \mathcal{M}_{g}$ and $P \in C$.

Applying Proposition A.3 to the case where $a=0$, and contracting all (-2)curves in all singular fibers, we have a stable fibred surface $f_{0}: X_{0} \rightarrow Y_{0}$ such that $Y_{0}$ is projective, the generic fiber of $f_{0}$ is a smooth hyperelliptic curve of genus $g$, $f_{0}$ is not smooth, and that every singular fiber of $f_{0}$ is an irreducible nodal curve with one node. Let $\mu_{0}: Y_{0} \rightarrow \overline{\mathcal{M}}_{g}$ be the induced morphism by $f_{0}: X_{0} \rightarrow Y_{0}$. Then, $C_{0}=\mu\left(Y_{0}\right)$ is our desired curve.

Finally, we fix $i$ with $1 \leq i \leq[g / 2]$. Using Proposition A.2, there is a stable fibred surface $f_{i}: X_{i} \rightarrow Y_{i}$ such that $Y_{i}$ is projective, the generic fiber of $f_{i}$ is a smooth hyperelliptic curve of genus $g, f_{i}$ is not smooth, and that every singular fiber of $f_{i}$ is a reducible curve with one node of type $i$. Let $\mu_{i}: Y_{i} \rightarrow \overline{\mathcal{M}}_{g}$ be the induced morphism by $f_{i}: X_{i} \rightarrow Y_{i}$. If we set $C_{i}=\mu_{i}\left(Y_{i}\right)$, then $C_{i}$ satisfies our requirements.

By using curves in Lemma 4.1, we can show the following proposition.

\section{Proposition 4.2.}

$$
\begin{aligned}
& \operatorname{Nef}\left(\overline{\mathcal{M}}_{g}, \operatorname{Cur}_{\mathcal{I}_{g}}^{\overline{\mathcal{M}}_{g}^{\text {one }}}\right) \\
& \quad \subseteq\left\{\begin{array}{l|l}
x \lambda+\sum_{i=0}^{[g / 2]} y_{i} \delta_{i} & \begin{array}{l}
x \geq 0 \\
g x+(8 g+4) y_{0} \geq 0 \\
i(g-i) x+(2 g+1) y_{i} \geq 0 \quad(1 \leq i \leq[g / 2])
\end{array}
\end{array}\right\} .
\end{aligned}
$$

Proof. Let $D=x \lambda+\sum_{i=0}^{[g / 2]} y_{i} \delta_{i}$ be an arbitrary element of $\operatorname{Nef}\left(\overline{\mathcal{M}}_{g}, \operatorname{Cur}_{\mathcal{I}_{g}}^{\overline{\mathcal{M}}_{g}^{\text {one }}}\right)$. Let $C, C_{0}, \ldots, C_{[g / 2]}$ be irreducible complete curves as in Lemma 4.1. Then, $0 \leq$ $(D \cdot C)=x(\lambda \cdot C)$. Hence $x \geq 0$.

To get other inequalities, we need some facts about hyperelliptic fibrations. Details can be found in $[3, \S 4, \mathrm{~b}]$. For $i>0, \Delta_{i} \cap \overline{\mathcal{I}}_{g}$ is irreducible. $\Delta_{0} \cap \overline{\mathcal{I}}_{g}$ is however reducible and has $1+[(g-1) / 2]$ irreducible components, say, $\Sigma_{0}, \Sigma_{1}, \ldots, \Sigma_{[(g-1) / 2]}$. Here a general point of $\Sigma_{0}$ represents an irreducible curve of one node, and a general point of $\Sigma_{i}(i>0)$ represents a stable curve consisting of a curve of genus $i$ and a curve of genus $g-i-1$ joined at two points. The class of $\Sigma_{i}$ in $\operatorname{Pic}\left(\overline{\mathcal{I}}_{g}\right) \otimes \mathbb{Q}$ is denoted by $\sigma_{i}$, and by abuse of notation, $\left.\delta_{i}\right|_{\overline{\mathcal{I}}_{g}}$ is denoted by $\delta_{i}$. Further, $\left.\lambda\right|_{\overline{\mathcal{I}}_{g}}$ is denoted by $\lambda$. Then, by virtue of [3, Proposition (4.7)],

$$
\delta_{0}=\sigma_{0}+2\left(\sigma_{1}+\cdots+\sigma_{[(g-1) / 2]}\right)
$$

and

$$
(8 g+4) \lambda=g \sigma_{0}+\sum_{j=1}^{[(g-1) / 2]} 2(j+1)(g-j) \sigma_{j}+\sum_{i=1}^{[g / 2]} 4 i(g-i) \delta_{i} .
$$


Let us view $D$ as a divisor on $\overline{\mathcal{I}}_{g}$. Using the above relations between $\lambda, \delta_{i}$ 's and $\sigma_{j}$ 's, we have

$$
\begin{aligned}
D=\left(\frac{g}{8 g+4} x+y_{0}\right) \sigma_{0}+2 & \sum_{j=1}^{[(g-1) / 2]}\left(\frac{(j+1)(g-j)}{8 g+4} x+y_{0}\right) \sigma_{j} \\
& +\sum_{i=1}^{[g / 2]}\left(\frac{i(g-i)}{2 g+1} x+y_{i}\right) \delta_{i} .
\end{aligned}
$$

Note that $C_{i} \cap \Sigma_{j}=\emptyset$ for all $0 \leq i \leq[g / 2]$ and $1 \leq j \leq[(g-1) / 2]$ because $C_{i} \subset \overline{\mathcal{M}}_{g}^{\text {one }}$. Thus, considering $\left(D \cdot C_{i}\right)$, we have the remaining inequalities.

Corollary 4.3. If $\mathcal{C}$ is a set of complete irreducible curves on $\overline{\mathcal{M}}_{g}$ with $\operatorname{Cur}_{\mathcal{I}_{g}}^{\overline{\mathcal{M}}_{g}^{\text {one }}} \subseteq$ $\mathcal{C} \subseteq \operatorname{Cur}_{\mathcal{M}_{g}}^{\overline{\mathcal{M}}_{g}}$, then

$\operatorname{Nef}\left(\overline{\mathcal{M}}_{g}, \mathcal{C}\right)=\left\{\begin{array}{l|l}x \lambda+\sum_{i=0}^{[g / 2]} y_{i} \delta_{i} & \begin{array}{l}x \geq 0 \\ g x+(8 g+4) y_{0} \geq 0 \\ i(g-i) x+(2 g+1) y_{i} \geq 0 \quad(1 \leq i \leq[g / 2])\end{array}\end{array}\right\}$.

Proof. Since $\operatorname{Nef}\left(\overline{\mathcal{M}}_{g}, \mathcal{C}\right) \subseteq \operatorname{Nef}\left(\overline{\mathcal{M}}_{g}, \operatorname{Cur}_{\mathcal{I}_{g}}^{{\overline{\mathcal{M}^{\prime}}}_{g}^{\text {one }}}\right)$, the direction " $\subseteq$ " is a consequence of Proposition 4.2. Conversely, we assume that $D=x \lambda+\sum_{i=0}^{[g / 2]} y_{i} \delta_{i}$ satisfies

$$
\left\{\begin{array}{l}
x \geq 0 \\
g x+(8 g+4) y_{0} \geq 0 \\
i(g-i) x+(2 g+1) y_{i} \geq 0 \quad(1 \leq i \leq[g / 2]) .
\end{array}\right.
$$

Then, since

$$
\begin{aligned}
D=\frac{x}{8 g+4}\left((8 g+4) \lambda-g \delta_{0}\right. & \left.-\sum_{i=1}^{[g / 2]} 4 i(g-i) \delta_{i}\right) \\
& +\left(y_{0}+\frac{g}{8 g+4} x\right) \delta_{0}+\sum_{i=1}^{[g / 2]}\left(y_{i}+\frac{i(g-i)}{2 g+1} x\right) \delta_{i}
\end{aligned}
$$

and $\mathcal{C} \subseteq \operatorname{Cur}_{\mathcal{M}_{g}}^{\overline{\mathcal{M}}_{g}}$, we can see that $D$ is numerically effective for $\mathcal{C}$ by using Theorem 3.2 .

In the same way, we can see the following.

Corollary 4.4. If we set

$$
\mathrm{WP}\left(\overline{\mathcal{M}}_{g} ; \mathcal{M}_{g}\right)=\left\{D \in \operatorname{Pic}\left(\overline{\mathcal{M}}_{g}\right) \otimes \mathbb{Q} \mid D \text { is weakly positive over } \mathcal{M}_{g}\right\}
$$

then

$$
\mathrm{WP}\left(\overline{\mathcal{M}}_{g} ; \mathcal{M}_{g}\right)=\left\{\begin{array}{l|l}
x \lambda+\sum_{i=0}^{[g / 2]} y_{i} \delta_{i} & \begin{array}{l}
x \geq 0 \\
g x+(8 g+4) y_{0} \geq 0 \\
i(g-i) x+(2 g+1) y_{i} \geq 0 \quad(1 \leq i \leq[g / 2])
\end{array}
\end{array}\right\} .
$$


Proof. Note that

$$
\mathrm{WP}\left(\overline{\mathcal{M}}_{g} ; \mathcal{M}_{g}\right) \subseteq \operatorname{Nef}\left(\overline{\mathcal{M}}_{g}, \operatorname{Cur}_{\mathcal{M}_{g}}^{\overline{\mathcal{M}}_{g}}\right)
$$

and that $(8 g+4) \lambda-g \delta_{0}-\sum_{i=1}^{[g / 2]} 4 i(g-i)$ and $\delta_{i}$ 's are weakly positive over $\mathcal{M}_{g}$.

Remark 4.5. In general, over an open set, weak positivity is stronger than numerical effectivity. Corollary 4.3 and Corollary 4.4 however say that, on the moduli space of stable curves $\overline{\mathcal{M}}_{g}$, weak positivity over $\mathcal{M}_{g}$ coincides with numerical effectivity over $\mathcal{M}_{g}$.

\section{EFFeCtive Bogomolov's CONJECTURE OVER FUnCtion FIELDS}

Let $X$ be a smooth projective surface over $k, Y$ a smooth projective curve over $k$, and $f: X \rightarrow Y$ a generically smooth semistable curve of genus $g \geq 2$ over $Y$. Let $K$ be the function field of $Y, \bar{K}$ the algebraic closure of $K$, and $C$ the generic fiber of $f$. Let $j: C(\bar{K}) \rightarrow \operatorname{Jac}(C)(\bar{K})$ be the map given by $j(x)=(2 g-2) x-\omega_{C}$, and let \|\|$_{N T}$ be the semi-norm arising from the Neron-Tate height pairing on $\operatorname{Jac}(C)(\bar{K})$. We set

$$
B_{C}(P ; r)=\left\{x \in C(\bar{K}) \mid\|j(x)-P\|_{N T} \leq r\right\}
$$

for $P \in \operatorname{Jac}(C)(\bar{K})$ and $r \geq 0$, and

$$
r_{C}(P)= \begin{cases}-\infty & \text { if } \#\left(B_{C}(P ; 0)\right)=\infty, \\ \sup \left\{r \geq 0 \mid \#\left(B_{C}(P ; r)\right)<\infty\right\} & \text { otherwise. }\end{cases}
$$

An effective version of Bogomolov's conjecture claims the following.

Conjecture 5.1 (Effective Bogomolov's conjecture). If $f$ is non-isotrivial, then there is an effectively calculated positive number $r_{0}$ with

$$
\inf _{P \in \operatorname{Jac}(C)(\bar{K})} r_{C}(P) \geq r_{0} .
$$

Recently, Ullmo [19] proved that $r_{C}(P)>0$ for all $P \in \operatorname{Jac}(C)(\bar{K})$ for the case where $K$ is a number field. As far as we know, the problem of finding an effectively calculated $r_{0}$ is still open. The meaning of "effectively calculated" is that a concrete algorithm or formula to find $r_{0}$ is required.

Here we need a rather technical condition coming from calculations of Green functions along singular fibers. Let $\bar{f}: \bar{X} \rightarrow Y$ be the stable model of $f: X \rightarrow Y$. Let $X_{y}$ (resp. $\bar{X}_{y}$ ) be the singular fiber of $f$ (resp. $\bar{f}$ ) over $y \in Y$, and let $S_{y}$ be the set of nodes $P$ on $\bar{X}_{y}$ such that $P$ is not an intersection of two irreducible components of $\bar{X}_{y}$, i.e., a singularity of an irreducible component. Let $\pi: Z_{y} \rightarrow \bar{X}_{y}$ be the partial normalization of $\bar{X}_{y}$ at each node in $S_{y}$. We say $X_{y}$ is a tree of stable components if the dual graph of $Z_{y}$ is a tree graph. In other words, every node of type 0 on $\bar{X}_{y}$ is a singularity of an irreducible component of $\bar{X}_{y}$.

As an application of Corollary 3.3, we get the following solution of the above conjecture, which is a generalization of [16, Theorem 5.2]. 
Theorem $5.2(\operatorname{char}(k)=0)$. If $f$ is not smooth and every singular fiber of $f$ is a tree of stable components, then

$$
\inf _{P \in \operatorname{Jac}(C)(\bar{K})} r_{C}(P) \geq \sqrt{\frac{(g-1)^{2}}{g(2 g+1)}\left(\frac{g-1}{3} \delta_{0}(X / Y)+\sum_{i=1}^{\left[\frac{g}{2}\right]} 4 i(g-i) \delta_{i}(X / Y)\right)} .
$$

Before starting the proof of Theorem 5.2, let us recall several facts about Green functions on a metrized graph. For details of metrized graphs, see [21].

Let $G$ be a connected metrized graph and $D$ an $\mathbb{R}$-divisor on $G$. If $\operatorname{deg}(D) \neq-2$, then there are a unique measure $\mu_{(G, D)}$ on $G$ and a unique function $g_{(G, D)}$ on $G \times G$ with the following properties.

(a) $\int_{G} \mu_{(G, D)}=1$

(b) $g_{(G, D)}(x, y)$ is symmetric and continuous on $G \times G$.

(c) For a fixed $x \in G, \Delta_{y}\left(g_{(G, D)}(x, y)\right)=\delta_{x}-\mu_{(G, D)}$.

(d) For a fixed $x \in G, \int_{G} g_{(G, D)}(x, y) \mu_{(G, D)}(y)=0$.

(e) $g_{(G, D)}(D, y)+g_{(G, D)}(y, y)$ is a constant for all $y \in G$.

The constant $g_{(G, D)}(D, y)+g_{(G, D)}(y, y)$ is denoted by $c(G, D)$. Further we set

$$
\epsilon(G, D)=2 \operatorname{deg}(D) c(G, D)-g_{(G, D)}(D, D) .
$$

We would like to calculate the invariant $\epsilon(G, D)$ for the metrized graph $G$ with the polarization $D$. First of all, let us see two examples, which will be elementary pieces for our calculations.

Example 5.3 (cf. [14, Lemma 3.2]). Let $C$ be a circle of length $l$ and $O$ a point on $C$. Then,

$$
g_{(C, 0)}(O, O)=\frac{l}{12} \quad \text { and } \quad \epsilon(C, 0)=0 .
$$

Example 5.4 (cf. [16, Lemma 4.4]). Let $G$ be a segment of length $l$, and $P$ and $Q$ terminal points of $G$. Let $a$ and $b$ be real numbers with $a+b \neq 0$, and $D$ an $\mathbb{R}$-divisor on $G$ given by $D=(2 a-1) P+(2 b-1) Q$. Then,

$$
\epsilon(G, D)=\left(\frac{4 a b}{a+b}-1\right) l, \quad g_{(G, D)}(P, P)=\frac{b^{2}}{(a+b)^{2}} l, \quad g_{(G, D)}(Q, Q)=\frac{a^{2}}{(a+b)^{2}} l .
$$

Let $G_{1}$ and $G_{2}$ be metrized graphs. Fix points $x_{1} \in G_{1}$ and $x_{2} \in G_{2}$. The one point sum $G_{1} \vee G_{2}$ with respect to $x_{1}$ and $x_{2}$, defined by $G_{1} \times\left\{x_{2}\right\} \cup\left\{x_{1}\right\} \times G_{2}$ in $G_{1} \times G_{2}$, is a metrized graph obtained by joining $x_{1} \in G_{1}$ and $x_{2} \in G_{2}$. The joining point, which is $\left\{x_{1}\right\} \times\left\{x_{2}\right\}$ in $G_{1} \times G_{2}$, is denoted by $j\left(G_{1} \vee G_{2}\right)$. Any $\mathbb{R}$-divisor on $G_{i}(i=1,2)$ can be viewed as an $\mathbb{R}$-divisor on $G_{1} \vee G_{2}$. Then, our basic tool for our calculations is the following.

Proposition 5.5 (cf. [16, Proposition 4.2]). Let $G_{1}$ and $G_{2}$ be connected metrized graphs, and $D_{1}$ and $D_{2} \mathbb{R}$-divisors on $G_{1}$ and $G_{2}$ respectively with $\operatorname{deg}\left(D_{i}\right) \neq-2$ $(i=1,2)$. Let $G=G_{1} \vee G_{2}, O=j\left(G_{1} \vee G_{2}\right)$, and $D=D_{1}+D_{2}$ on $G_{1} \vee G_{2}$. If $\operatorname{deg}\left(D_{1}+D_{2}\right) \neq-2$, then we have the following formulae, where $d_{i}=\operatorname{deg}\left(D_{i}\right)$ $(i=1,2)$ and $r_{G_{2}}(O, P)$ is the resistance between $O$ and $P$ on $G_{2}$. 
(1) If $P \in G_{2}$, then

$$
\begin{aligned}
g_{(G, D)}(P, P)= & \frac{d_{1}}{d_{1}+d_{2}+2} r_{G_{2}}(O, P)+\frac{d_{2}+2}{d_{1}+d_{2}+2} g_{\left(G_{2}, D_{2}\right)}(P, P) \\
& -\frac{d_{1}\left(d_{2}+2\right)}{\left(d_{1}+d_{2}+2\right)^{2}} g_{\left(G_{2}, D_{2}\right)}(O, O)+\frac{\left(d_{1}+2\right)^{2}}{\left(d_{1}+d_{2}+2\right)^{2}} g_{\left(G_{1}, D_{1}\right)}(O, O) .
\end{aligned}
$$

$$
\begin{aligned}
\epsilon(G, D)=\epsilon\left(G_{1}, D_{1}\right) & +\epsilon\left(G_{2}, D_{2}\right) \\
& +\frac{2 d_{2}\left(d_{1}+2\right) g_{\left(G_{1}, D_{1}\right)}(O, O)+2 d_{1}\left(d_{2}+2\right) g_{\left(G_{2}, D_{2}\right)}(O, O)}{d_{1}+d_{2}+2} .
\end{aligned}
$$

Combining the above proposition and Example 5.3, we have the following.

Corollary 5.6. Let $G$ be a connected metrized graph and $D$ an $\mathbb{R}$-divisor on $G$ with $\operatorname{deg}(D) \neq-2$. Let $C$ be a circle of length l. Then,

$$
\epsilon(G \vee C, D)=\epsilon(G, D)+\frac{\operatorname{deg}(D)}{3(\operatorname{deg} D+2)} l .
$$

Let $G$ be a connected metrized graph. We assume that $G$ is a tree, i.e., there is no loop in $G$. Let $\operatorname{Vert}(G)($ resp. $\operatorname{Ed}(G))$ be the set of vertices (resp. edges) of $G$. For a function $\alpha: \operatorname{Vert}(G) \rightarrow \mathbb{R}$, we define the divisor $D(\alpha)$ on $G$ to be

$$
D(\alpha)=\sum_{x \in \operatorname{Vert}(G)}(2 \alpha(x)-2+v(x)) x,
$$

where $v(x)$ is the number of branches starting from $x$. It is easy to see that

$$
\operatorname{deg}(D(\alpha))+2=2 \sum_{x \in \operatorname{Vert}(G)} \alpha(x)
$$

To give an exact formula for $\epsilon(G, D(\alpha))$, we need to introduce the following notation. Let $e$ be an edge of $G, P$ and $Q$ terminal points of $e$, and $e^{\circ}=e \backslash\{P, Q\}$. Since $G$ is a connected tree, there are two connected sub-graphs $G_{e}^{\prime}$ and $G_{e}^{\prime \prime}$ such that $G \backslash e^{\circ}=G_{e}^{\prime} \amalg G_{e}^{\prime \prime}$. Then, we have the following.

Proposition 5.7. With the same notation as above, if $\alpha(x) \geq 0$ for all $x \in \operatorname{Vert}(G)$ and $\sum_{x \in \operatorname{Vert}(G)} \alpha(x) \neq 0$, then

$$
\epsilon(G, D(\alpha))=\sum_{e \in \operatorname{Ed}(G)}\left(\frac{4\left(\sum_{x \in \operatorname{Vert}\left(G_{e}^{\prime}\right)} \alpha(x)\right)\left(\sum_{x \in \operatorname{Vert}\left(G_{e}^{\prime \prime}\right)} \alpha(x)\right)}{\sum_{x \in \operatorname{Vert}(G)} \alpha(x)}-1\right) l(e),
$$

where $l(e)$ is the length of $e$.

Proof. For a positive number $t$, we set $\alpha_{t}(x)=\alpha(x)+t$. Then, it is easy to see that

$$
\lim _{t \downarrow 0} \epsilon\left(G, D\left(\alpha_{t}\right)\right)=\epsilon(G, D(\alpha)) .
$$

Thus, in order to prove our proposition, we may assume that $\alpha(x)>0$ for all $x \in \operatorname{Vert}(G)$.

We fix $P \in \operatorname{Vert}(G)$. For $e \in \operatorname{Ed}(G)$, we denote by $G_{P, e}$ the connected component of $G \backslash e^{\circ}$ not containing $P$, i.e., if $P \notin G_{e}^{\prime}$, then $G_{P, e}=G_{e}^{\prime}$; otherwise, $G_{P, e}=G_{e}^{\prime \prime}$. With this notation, let us consider the following claim. 
Claim 5.7.1.

$$
g_{(G, D(\alpha))}(P, P)=\sum_{e \in \operatorname{Ed}(G)} \frac{\left(\sum_{x \in \operatorname{Vert}\left(G_{P, e}\right)} \alpha(x)\right)^{2}}{\left(\sum_{x \in \operatorname{Vert}(G)} \alpha(x)\right)^{2}} l(e) .
$$

We prove this claim by induction on $\#(\operatorname{Ed}(G))$. If $\#(\operatorname{Ed}(G))=0,1$, then our assertion is obvious by Example 5.4. Thus, we may assume that $\#(\operatorname{Ed}(G)) \geq 2$.

First, we suppose that $P$ is not a terminal point. Let $G^{\prime}$ be one branch starting from $P$, and $G^{\prime \prime}$ a connected sub-graph such that $G^{\prime} \cup G^{\prime \prime}=G$ and $G^{\prime} \cap G^{\prime \prime}=\{P\}$. We define $\alpha^{\prime}: \operatorname{Vert}\left(G^{\prime}\right) \rightarrow \mathbb{R}$ and $\alpha^{\prime \prime}: \operatorname{Vert}\left(G^{\prime \prime}\right) \rightarrow \mathbb{R}$ by

$$
\alpha^{\prime}(x)= \begin{cases}1 & \text { if } x=P, \\ \alpha(x) & \text { otherwise }\end{cases}
$$

and $\alpha^{\prime \prime}=\left.\alpha\right|_{\operatorname{Vert}\left(G^{\prime \prime}\right)}$. Then, we have $G=G^{\prime} \vee G^{\prime \prime}$ and $D(\alpha)=D\left(\alpha^{\prime}\right)+D\left(\alpha^{\prime \prime}\right)$. Thus, using (1) of Proposition 5.5 and the hypothesis of induction, we can easily see our claim.

Next we suppose that $P$ is a terminal point. Pick up $e \in \operatorname{Ed}(G)$ such that $P$ is a terminal of $e$. Let $O$ be another terminal of $e$. We set $G^{\prime}=e$ and $G^{\prime \prime}=(G \backslash e) \cup\{O\}$. Moreover, we define $\alpha^{\prime}: \operatorname{Vert}\left(G^{\prime}\right)=\{P, O\} \rightarrow \mathbb{R}$ and $\alpha^{\prime \prime}: \operatorname{Vert}\left(G^{\prime \prime}\right) \rightarrow \mathbb{R}$ by $\alpha^{\prime}(P)=\alpha(P), \alpha^{\prime}(O)=1$ and $\alpha^{\prime \prime}=\left.\alpha\right|_{\operatorname{Vert}\left(G^{\prime \prime}\right)}$. Then, $G=G^{\prime} \vee G^{\prime \prime}$ and $D(\alpha)=$ $D\left(\alpha^{\prime}\right)+D\left(\alpha^{\prime \prime}\right)$. Thus, using (1) of Proposition 5.5, Example 5.4 and the hypothesis of induction, we can see our claim after easy calculations.

Let us go back to the proof of Proposition 5.7. We prove it by induction on $\#(\operatorname{Ed}(G))$. If $\#(\operatorname{Ed}(G))=0,1$, then our assertion comes from Example 5.4. Thus, we may assume that $\#(\operatorname{Ed}(G)) \geq 2$. Let us pick up a terminal edge $e$ of $G$. Let $\{O, P\}$ be terminals of $e$ such that $P$ gives a terminal of $G$. We set $G_{1}=e$ and $G_{2}=(G \backslash e) \cup\{O\}$. Moreover, we define $\alpha_{1}:\{O, P\}=\operatorname{Vert}\left(G_{1}\right) \rightarrow \mathbb{R}$ and $\alpha_{2}: \operatorname{Vert}\left(G_{2}\right) \rightarrow \mathbb{R}$ by $\alpha_{1}(O)=1, \alpha_{1}(P)=\alpha(P)$, and $\alpha_{2}=\left.\alpha\right|_{\operatorname{Vert}\left(G_{2}\right)}$. Then, $G=G_{1} \vee G_{2}$ and $D(\alpha)=D\left(\alpha_{1}\right)+D\left(\alpha_{2}\right)$. Thus, if we set

$$
\left\{\begin{array}{l}
A=\sum_{x \in \operatorname{Vert}(G)} \alpha(x), \\
a=\alpha(P), \\
A_{e^{\prime}}=\sum_{x \in \operatorname{Vert}\left(G_{O, e^{\prime}}\right)} \alpha(x) \quad \text { for } e^{\prime} \in \operatorname{Vert}(G) \backslash\{e\},
\end{array}\right.
$$

then, by (2) of Proposition 5.5, Example 5.4, Claim 5.7.1 and the hypothesis of induction, we have

$$
\begin{aligned}
\epsilon(G, D(\alpha))= & \left(\frac{4 a}{a+1}-1\right) l(e)+\sum_{e^{\prime} \in \operatorname{Vert}(G) \backslash\{e\}}\left(\frac{4 A_{e^{\prime}}\left(A-a-A_{e^{\prime}}\right)}{A-a}-1\right) l\left(e^{\prime}\right) \\
& +\frac{4(A-a-1)(a+1)}{A} \frac{a^{2}}{(a+1)^{2}} l(e) \\
& +\frac{4 a(A-a)}{A} \sum_{e^{\prime} \in \operatorname{Vert}(G) \backslash\{e\}} \frac{A_{e^{\prime}}^{2}}{(A-a)^{2}} l\left(e^{\prime}\right) \\
= & \left(\frac{4 a(A-a)}{A}-1\right) l(e)+\sum_{e^{\prime} \in \operatorname{Vert}(G) \backslash\{e\}}\left(\frac{4 A_{e^{\prime}}\left(A-A_{e^{\prime}}\right)}{A}-1\right) l\left(e^{\prime}\right) .
\end{aligned}
$$

Therefore, we get our proposition. 
Corollary $5.8(\operatorname{char}(k) \geq 0)$. Let $X$ be a smooth projective surface over $k, Y$ a smooth projective curve over $k$, and $f: X \rightarrow Y$ a generically smooth semistable curve of genus $g \geq 2$ over $Y$. Let $X_{y}$ be the singular fiber of $f$ over $y \in Y$, and $X_{y}=C_{1}+\cdots+C_{n}$ the irreducible decomposition of $X_{y}$. Let $G_{y}$ be the metrized graph given by the configuration of $X_{y}, v_{i}$ the vertex of $G_{y}$ corresponding to $C_{i}$, and $\omega_{y}$ the divisor on $G_{y}$ defined by $\omega_{y}=\sum_{i}\left(\omega_{X / Y} \cdot C_{i}\right) v_{i}$. If $X_{y}$ is a tree of stable components, then

$$
\epsilon\left(G_{y}, \omega_{y}\right)=\frac{g-1}{3 g} \delta_{0}\left(X_{y}\right)+\sum_{i=1}^{\left[\frac{g}{2}\right]}\left(\frac{4 i(g-i)}{g}-1\right) \delta_{i}\left(X_{y}\right) .
$$

Proof. Let $\bar{f}: \bar{X} \rightarrow Y$ be the stable model of $f: X \rightarrow Y$, and let $S_{y}$ be the set of nodes $P$ on $\bar{X}_{y}$ such that $P$ is a singularity of an irreducible component. Let $\pi: Z_{y} \rightarrow \bar{X}_{y}$ be the partial normalization of $\bar{X}_{y}$ at each node in $S_{y}$. Let $\bar{G}_{y}$ be the dual graph of $Z_{y}$. Let $l_{1}, \ldots, l_{r}$ be circles in $G_{y}$ corresponding to nodes in $S_{y}$. Then, $G_{y}=\bar{G}_{y} \vee l_{1} \vee \cdots \vee l_{r}$. Moreover, if $g_{i}$ is the arithmetic genus of $C_{i}$ and $\alpha: \operatorname{Vert}\left(G_{y}\right) \rightarrow \mathbb{R}$ is given by $\alpha\left(v_{i}\right)=g_{i}$, then $\omega_{y}=D(\alpha)$. Here, by virtue of Proposition 5.7,

$$
\epsilon\left(\bar{G}_{y}, \omega_{y}\right)=\sum_{i=1}^{\left[\frac{g}{2}\right]}\left(\frac{4 i(g-i)}{g}-1\right) \delta_{i}\left(X_{y}\right) .
$$

Therefore, it follows from Corollary 5.6 that

$$
\epsilon\left(G_{y}, \omega_{y}\right)=\frac{g-1}{3 g} \delta_{0}\left(X_{y}\right)+\sum_{i=1}^{\left[\frac{g}{2}\right]}\left(\frac{4 i(g-i)}{g}-1\right) \delta_{i}\left(X_{y}\right) .
$$

Proof of Theorem 5.2. First of all, note the following fact (cf. [21, Theorem 5.6], $\left[14\right.$, Corollary 2.3] or $\left[15\right.$, Theorem 2.1]). If $\left(\omega_{X / Y}^{a} \cdot \omega_{X / Y}^{a}\right)_{a}>0$, then

$$
\inf _{P \in \operatorname{Jac}(C)(\bar{K})} r_{C}(P) \geq \sqrt{(g-1)\left(\omega_{X / Y}^{a} \cdot \omega_{X / Y}^{a}\right)_{a}},
$$

where $(\cdot)_{a}$ is the admissible pairing.

By the definition of admissible pairing, we can see

$$
\left(\omega_{X / Y}^{a} \cdot \omega_{X / Y}^{a}\right)_{a}=\left(\omega_{X / Y} \cdot \omega_{X / Y}\right)-\sum_{y \in Y} \epsilon\left(G_{y}, \omega_{y}\right) .
$$

On the other hand, by Corollary 3.3, we have

$$
(8 g+4) \operatorname{deg}\left(f_{*}\left(\omega_{X / Y}\right)\right) \geq g \delta_{0}(X / Y)+\sum_{i=1}^{\left[\frac{g}{2}\right]} 4 i(g-i) \delta_{i}(X / Y) .
$$

Thus, using Noether's formula, the above inequality implies

$$
\left(\omega_{X / Y} \cdot \omega_{X / Y}\right) \geq \frac{g-1}{2 g+1} \delta_{0}(X / Y)+\sum_{i=1}^{\left[\frac{g}{2}\right]}\left(\frac{12 i(g-i)}{2 g+1}-1\right) \delta_{i}(X / Y) .
$$

Therefore, we have our theorem by Corollary 5.8 . 
Moreover, using Ullmo's result [19] and Proposition 5.7, we have the following.

Corollary 5.9. Let $K$ be a number field, $O_{K}$ the ring of integers, and $f: X \rightarrow$ $\operatorname{Spec}\left(O_{K}\right)$ a regular semistable arithmetic surface of genus $g \geq 2$ over $O_{K}$. Let $S$ be the subset of $\operatorname{Spec}\left(O_{K}\right) \backslash\{0\}$ such that $P \in S$ if and only if the stable model of the geometric fiber $X_{\bar{P}}$ at $P$ is a tree of stable components. Then, we have

$$
\begin{aligned}
& \left(\omega_{X / O_{K}}^{A r} \cdot \omega_{X / O_{K}}^{A r}\right) \\
& \quad>\sum_{P \in S}\left\{\frac{g-1}{3 g} \delta_{0}\left(X_{\bar{P}}\right)+\sum_{i=1}^{[g / 2]}\left(\frac{4 i(g-i)}{g}-1\right) \delta_{i}\left(X_{\bar{P}}\right)\right\} \log \#\left(O_{K} / P\right) .
\end{aligned}
$$

\section{Generalization to higher dimensional fibrations}

In this section, we consider a generalization of Corollary 2.5 to higher dimensional fibrations.

First of all, let us recall the definition of semistability of vector bundles. Let $V$ be a smooth projective variety of dimension $d$ over $k$, and let $H_{1}, \ldots, H_{d-1}$ be ample line bundles on $V$. A vector bundle $E$ on $V$ is said to be semistable with respect to $H_{1}, \ldots, H_{d-1}$ if, for any non-zero subsheaves $G$ of $E$,

$$
\frac{\left(c_{1}(G) \cdot c_{1}\left(H_{1}\right) \cdots c_{1}\left(H_{d-1}\right)\right)}{\operatorname{rk} G} \leq \frac{\left(c_{1}(E) \cdot c_{1}\left(H_{1}\right) \cdots c_{1}\left(H_{d-1}\right)\right)}{\operatorname{rk} E} .
$$

Let $f: X \rightarrow Y$ be a surjective and projective morphism of quasi-projective varieties over $k$ with $\operatorname{dim} f=d \geq 1$. Let $H_{1}, \ldots, H_{d-1}$ be line bundles on $X$ and $E$ a vector bundle of rank $r$ on $X$. Then,

$$
\left(\left(2 r c_{2}(E)-(r-1) c_{1}(E)^{2}\right) \cdot c_{1}\left(H_{1}\right) \cdots c_{1}\left(H_{d-1}\right)\right) \cap[X]
$$

is a cycle of dimension $\operatorname{dim} Y-1$ on $X$. Thus,

$$
f_{*}\left(\left(\left(2 r c_{2}(E)-(r-1) c_{1}(E)^{2}\right) \cdot c_{1}\left(H_{1}\right) \cdots c_{1}\left(H_{d-1}\right)\right) \cap[X]\right),
$$

denoted by $\operatorname{dis}_{X / Y}\left(E ; H_{1}, \ldots, H_{d-1}\right)$, is a divisor on $Y$. Then, we have the following theorem.

Theorem $6.1(\operatorname{char}(k)=0)$. We assume that $Y$ is smooth over $k$ and $H_{1}, \ldots$, $H_{d-1}$ are ample. If $y$ is a point of $Y, f$ is smooth over $y$, and $E_{\bar{y}}$ is semistable with respect to $\left(H_{1}\right)_{\bar{y}}, \ldots,\left(H_{d-1}\right)_{\bar{y}}$ on each connected component of the geometric fiber $X_{\bar{y}}$ over $y$, then the discriminant divisor $\operatorname{dis}_{X / Y}\left(E ; H_{1}, \ldots, H_{d-1}\right)$ is weakly positive at $y$.

Proof. We prove this theorem by induction on $d$. If $d=1$, then our assertion is nothing more than Corollary 2.5. So we assume $d \geq 2$. We choose a sufficiently large integer $n$ so that $H_{d-1}^{\otimes n}$ is very ample, i.e., there is an embedding $\iota: X \hookrightarrow \mathbb{P}^{N}$ with $H_{d-1}^{\otimes n} \simeq \iota^{*}\left(\mathcal{O}_{\mathbb{P}^{N}}(1)\right)$. By Bertini's theorem, we can find a general member $\Gamma \in\left|\mathcal{O}_{\mathbb{P}^{N}}(1)\right|$ such that $X \cap \Gamma$ is integral and $f^{-1}(y) \cap \Gamma$ is smooth. We set $Z=X \cap \Gamma$ and $g=\left.f\right|_{Z}: Z \rightarrow Y$. Since $n$ is sufficiently large, $g^{-1}(y) \in\left|H_{d-1}^{\otimes n}\right|_{f^{-1}(y)} \mid$ and $g^{-1}(y)$ is smooth, by virtue of [13, Theorem 3.1], $\left.E\right|_{Z_{\bar{y}}}$ is semistable with respect to $\left.H_{1}\right|_{Z_{\bar{y}}}, \ldots,\left.H_{d-2}\right|_{Z_{\bar{y}}}$ on each connected component of $Z_{\bar{y}}$. Therefore, by the 
hypothesis of induction, $\operatorname{dis}_{Z / Y}\left(\left.E\right|_{Z} ;\left.H_{1}\right|_{Z}, \ldots,\left.H_{d-2}\right|_{Z}\right)$ is weakly positive at $y$. On the other hand, we have

$$
\begin{aligned}
\operatorname{dis}_{Z / Y}\left(\left.E\right|_{Z} ;\left.H_{1}\right|_{Z}, \ldots,\left.H_{d-2}\right|_{Z}\right) & =\operatorname{dis}_{X / Y}\left(E ; H_{1}, \ldots, H_{d-2}, H_{d-1}^{\otimes n}\right) \\
& =n \operatorname{dis}_{X / Y}\left(E ; H_{1}, \ldots, H_{d-1}\right) .
\end{aligned}
$$

Hence, $\operatorname{dis}_{X / Y}\left(E ; H_{1}, \ldots, H_{d-1}\right)$ is weakly positive at $y$.

\section{Relative Bogomolov's inequality in positive Characteristic}

In this section, we will consider a result similar to Corollary 2.5 in positive characteristic. The crucial point of the proof of Corollary 2.5 is the semistability of tensor products of semistable vector bundles, which was studied by many authors [7], [8], [12], [11], etc. This however does not hold in positive characteristic [6], so that we will introduce the strong semistability of vector bundles.

Let $C$ be a smooth projective curve over $k$. For a vector bundle $F$ on $C$, we set $\mu(F)=\operatorname{deg}(F) / \operatorname{rk}(F)$, which is called the slope of $F$. A vector bundle $E$ on $C$ is said to be semistable (resp. stable) if, for any proper subbundles $F$ of $E$, $\mu(F) \leq \mu(E)$ (resp. $\mu(F)<\mu(E)$ ). Moreover, $E$ is said to be strongly semistable if, for any finite morphisms $\pi: C^{\prime} \rightarrow C$ of smooth projective curves over $k, \pi^{*}(E)$ is semistable. Then, we have the following elementary properties of semistable or strongly semistable vector bundles.

Proposition $7.1(\operatorname{char}(k) \geq 0)$. Let $E$ be a vector bundle of rank $r$ on $C$.

(1) Let $\pi: C^{\prime} \rightarrow C$ be a finite separable morphism of smooth projective curves over $k$. If $E$ is semistable, then so is $\pi^{*}(E)$.

(2) Under the assumption of $\operatorname{char}(k)=0, E$ is semistable if and only if $E$ is strongly semistable.

(3) Let $f: P=\operatorname{Proj}\left(\bigoplus_{m=0}^{\infty} \operatorname{Sym}^{m}(E)\right) \rightarrow C$ be the projective bundle of $E$ and $\mathcal{O}_{P}(1)$ the tautological line bundle on $P$. Then, $E$ is strongly semistable if and only if $\omega_{P / C}^{\otimes-1}=\mathcal{O}_{P}(r) \otimes f^{*}(\operatorname{det} E)^{\otimes-1}$ is numerically effective.

Proof. (1) is nothing more than [7, Lemma 1.1] and (2) is a consequence of (1).

(3) First we assume that $E$ is strongly semistable. Let $Z$ be any irreducible curves on $P$. If $Z$ is contained in a fiber, then obviously $\left(\omega_{P / C}^{\otimes-1} \cdot Z\right)>0$. So we may assume that $Z$ is not contained in any fibers. Let $C^{\prime}$ be the normalization of $Z$ and $\pi: C^{\prime} \rightarrow Z \rightarrow C$ the induced morphism. Let $E^{\prime}=\pi^{*}(E)$, $f^{\prime}: P^{\prime}=\operatorname{Proj}\left(\bigoplus_{m=0}^{\infty} \operatorname{Sym}^{m}\left(E^{\prime}\right)\right) \rightarrow C^{\prime}$ the projective bundle of $E^{\prime}$, and $\mathcal{O}_{P^{\prime}}(1)$ the tautological line bundle on $P^{\prime}$. Then we have the following commutative diagram:

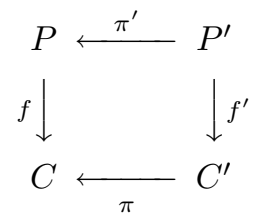

By our construction, there is a section $Z^{\prime}$ of $f^{\prime}$ such that $\pi^{\prime}\left(Z^{\prime}\right)=Z$. We set $Q^{\prime}=\left.\mathcal{O}_{P^{\prime}}(1)\right|_{Z^{\prime}}$. Then, there is a surjective homomorphism $E^{\prime} \rightarrow Q^{\prime}$. Since $E^{\prime}$ is semistable, we have $\mu\left(E^{\prime}\right) \leq \operatorname{deg}\left(Q^{\prime}\right)$, which means that $\left(\omega_{P^{\prime} / C^{\prime}}^{\otimes-1} \cdot Z^{\prime}\right) \geq 0$. Here, $\omega_{P^{\prime} / C^{\prime}}=\pi^{\prime *}\left(\omega_{P / C}\right)$. Thus, we get $\left(\omega_{P / C}^{\otimes-1} \cdot Z\right) \geq 0$. 
Conversely, we assume that $\omega_{P / C}^{\otimes-1}$ is numerically effective on $P$. Let $\pi: C^{\prime} \rightarrow C$ be a finite morphism of smooth projective curves over $k$. We set $f^{\prime}: P^{\prime} \rightarrow C^{\prime}$ and $\pi^{\prime}: P^{\prime} \rightarrow P$ as before. Then, $\omega_{P^{\prime} / C^{\prime}}^{\otimes-1}=\pi^{\prime *}\left(\omega_{P / C}^{\otimes-1}\right)$ is numerically effective on $P^{\prime}$. Let $Q$ be a quotient vector bundle of $E^{\prime}=\pi^{*}(E)$ with $s=\operatorname{rk} Q$. The projective bundle $\operatorname{Proj}\left(\bigoplus_{m=0}^{\infty} \operatorname{Sym}^{m}(Q)\right) \rightarrow C^{\prime}$ gives a subvariety $V^{\prime}$ of $P^{\prime}$ with $\operatorname{deg}(Q)=\left(\mathcal{O}_{P^{\prime}}(1)^{s} \cdot V^{\prime}\right)$ and $\left(\mathcal{O}_{P^{\prime}}(1)^{s-1} \cdot F^{\prime} \cdot V^{\prime}\right)=1$, where $F^{\prime}$ is a fiber of $f^{\prime}$. Since $\omega_{P^{\prime} / C^{\prime}}^{\otimes-1}$ is numerically effective,

$$
0 \leq\left(\left(\mathcal{O}_{P^{\prime}}(r) \otimes f^{\prime *}\left(\operatorname{det} E^{\prime}\right)^{-1}\right)^{s} \cdot V^{\prime}\right)=r^{s-1}\left(r \operatorname{deg}(Q)-s \operatorname{deg}\left(E^{\prime}\right)\right) .
$$

Thus, $\mu\left(E^{\prime}\right) \leq \mu(Q)$.

First, let us consider symmetric products of strongly semistable vector bundles.

Theorem $7.2(\operatorname{char}(k) \geq 0)$. If $E$ is a strongly semistable vector bundle on $C$, then so is $\operatorname{Sym}^{n}(E)$ for all $n \geq 0$.

Proof. Taking a finite covering of $C$, we may assume that $\operatorname{deg}(E)$ is divisible by rk $E$. Let $\theta$ be a line bundle on $C$ with $\operatorname{deg}(\theta)=1$. If we set $E_{0}=E \otimes \theta^{\otimes-\frac{\operatorname{deg}(E)}{\mathrm{rk} E}}$, then $\operatorname{deg}\left(E_{0}\right)=0$ and $\operatorname{Sym}^{n}\left(E_{0}\right)=\operatorname{Sym}^{n}(E) \otimes \theta^{\otimes-\frac{n \operatorname{deg}(E)}{\mathrm{rk} E}}$. Thus, to prove our theorem, we may assume $\operatorname{deg}(E)=0$.

We assume that $\operatorname{Sym}^{n}(E)$ is not strongly semistable for some $n \geq 2$. By replacing $C$ by a finite covering of $C$, we may assume that $\operatorname{Sym}^{n}(E)$ is not semistable. Let $f: P=\operatorname{Proj}\left(\bigoplus_{n=0}^{\infty} \operatorname{Sym}^{n}(E)\right) \rightarrow C$ be a projective bundle of $E$ and $\mathcal{O}_{P}(1)$ the tautological line bundle on $P$. Let $F$ be the maximal destabilizing sheaf of $\operatorname{Sym}^{n}(E)$. In particular, $F$ is semistable and $\mu(F)>0$. We consider a composition of homomorphisms

$$
\alpha: f^{*}(F) \rightarrow f^{*}\left(\operatorname{Sym}^{n}(E)\right) \rightarrow \mathcal{O}_{P}(n) .
$$

Since $f_{*}(\alpha)$ induces the inclusion $F \rightarrow \operatorname{Sym}^{n}(E), \alpha$ is a non-trivial homomorphism.

Fix an ample line bundle $A$ on $C$. Let $l$ be a positive integer with $l \mu(F)>$ $n(r-1) \operatorname{deg}(A)$ and $(l, p)=1$, where $p=\operatorname{char}(k)$. Here we claim that $\mathcal{O}_{P}(l) \otimes f^{*}(A)$ is ample. Let $V$ be an $s$-dimensional subvariety of $P$. By virtue of Nakai's criterion, it is sufficient to show $\left(c_{1}\left(\mathcal{O}_{P}(l) \otimes f^{*}(A)\right)^{s} \cdot V\right)>0$. If $V$ is contained in a fiber, our assertion is trivial. So we may assume that $V$ is not contained in any fibers. Then,

$$
\begin{aligned}
\left(c_{1}\left(\mathcal{O}_{P}(l) \otimes f^{*}(A)\right)^{s} \cdot V\right)=l^{s}\left(c_{1}\left(\mathcal{O}_{P}(1)\right)^{s} \cdot V\right) & \\
& +s l^{s-1}\left(c_{1}\left(\mathcal{O}_{P}(1)\right)^{s-1} \cdot c_{1}\left(f^{*}(A)\right) \cdot V\right) .
\end{aligned}
$$

Since $\mathcal{O}_{P}(1)$ is numerically effective on $P$ by (3) of Proposition 7.1, it follows that $\left(c_{1}\left(\mathcal{O}_{P}(1)\right)^{s} \cdot V\right) \geq 0$. Moreover, if $x$ is a general point of $C$,

$$
\left(c_{1}\left(\mathcal{O}_{P}(1)\right)^{s-1} \cdot c_{1}\left(f^{*}(A)\right) \cdot V\right)=\operatorname{deg}(A) \operatorname{deg}\left(\left.V\right|_{f^{-1}(x)}\right)>0 .
$$

Therefore, we get our claim.

Thus, there is a positive integer $m$ such that $\left(\mathcal{O}_{P}(l) \otimes f^{*}(A)\right)^{\otimes m}$ is very ample and $(m, p)=1$. Take general elements $D_{1}, \ldots, D_{r-1}$ of $\left|\left(\mathcal{O}_{P}(l) \otimes f^{*}(A)\right)^{\otimes m}\right|$ such that $\Gamma=D_{1} \cap \cdots \cap D_{r-1}$ is a non-singular curve and $\left.\left.f^{*}(F)\right|_{\Gamma} \rightarrow \mathcal{O}_{P}(n)\right|_{\Gamma}$ is generically surjective. If $x$ is a general point of $C$,

$$
\begin{aligned}
\operatorname{deg}(\Gamma \rightarrow C) & =\left(D_{1} \cdots D_{r-1} \cdot f^{-1}(x)\right) \\
& =m^{r-1}\left(c_{1}\left(\mathcal{O}_{P}(l) \otimes f^{*}(A)\right)^{r-1} \cdot f^{-1}(x)\right)=(m l)^{r-1} .
\end{aligned}
$$


Thus, $k(\Gamma)$ is separable over $k(C)$ because $\left(p,(m l)^{r-1}\right)=1$. Hence, $\left.f^{*}(F)\right|_{\Gamma}$ is semistable by (1) of Proposition 7.1. Therefore,

$$
\frac{\left(c_{1}\left(f^{*}(F)\right) \cdot \Gamma\right)}{\operatorname{rk} F} \leq\left(c_{1}\left(\mathcal{O}_{P}(n)\right) \cdot \Gamma\right),
$$

which implies

$$
\frac{\left(c_{1}\left(f^{*}(F)\right) \cdot c_{1}\left(\mathcal{O}_{P}(l) \otimes f^{*}(A)\right)^{r-1}\right)}{\operatorname{rk} F} \leq\left(c_{1}\left(\mathcal{O}_{P}(n)\right) \cdot c_{1}\left(\mathcal{O}_{P}(l) \otimes f^{*}(A)\right)^{r-1}\right) .
$$

This gives rise to

$$
l^{r-1} \mu(F) \leq n(r-1) l^{r-2} \operatorname{deg}(A),
$$

which contradicts the choice of $l$ with $l \mu(F)>n(r-1) \operatorname{deg}(A)$.

As a corollary of Theorem 7.2, we have the following.

Corollary $7.3(\operatorname{char}(k) \geq 0)$. If $E$ and $F$ are strongly semistable vector bundles on $C$, then so is $E \otimes F$.

Proof. Considering a finite covering of $C$ and tensoring line bundles, we may assume that $\operatorname{deg}(E)=\operatorname{deg}(F)=0$ in the same way as in the beginning part of the proof of Theorem 7.2. Then, $E \oplus F$ is strongly semistable. Thus, by Theorem 7.2, $\operatorname{Sym}^{2}(E \oplus F)$ is strongly semistable. Here,

$$
\operatorname{Sym}^{2}(E \oplus F)=(E \otimes F) \oplus \operatorname{Sym}^{2}(E) \oplus \operatorname{Sym}^{2}(F) .
$$

Therefore, we can see that $E \otimes F$ is strongly semistable.

Thus, in the same way as in the proof of Corollary 2.5, we have the following.

Corollary $7.4(\operatorname{char}(k) \geq 0)$. Let $X$ be a quasi-projective variety over $k, Y a$ smooth quasi-projective variety over $k$, and $f: X \rightarrow Y$ a surjective and projective morphism over $k$ with $\operatorname{dim} f=1$. Let $E$ be a locally free sheaf on $X$ and $y$ a point of $Y$. If $f$ is flat over $y$, the geometric fiber $X_{\bar{y}}$ over $y$ is reduced and Gorenstein, and $E$ is strongly semistable on each connected component of the normalization of $X_{\bar{y}}$, then $\operatorname{dis}_{X / Y}(E)$ is weakly positive at $y$.

\section{Appendix A. A CERTAin Fibration OF HYPERELliPtic CURVES}

In this section, we would like to construct a certain fibration of hyperelliptic curves, which is needed in $\S 4$. Throughout this section, we assume that $\operatorname{char}(k)=0$.

Let us begin with the following lemma.

Lemma A.1. For non-negative integers $a_{1}$ and $a_{2}$, there are a morphism $f_{1}$ : $X_{1} \rightarrow Y_{1}$ of smooth projective varieties over $k$, an effective divisor $D_{1}$ on $X_{1}$, a line bundle $L_{1}$ on $X_{1}$, and a line bundle $M_{1}$ on $Y_{1}$ with the following properties.

(1) $\operatorname{dim} X_{1}=2$ and $\operatorname{dim} Y_{1}=1$.

(2) Let $\Sigma_{1}$ be the set of all critical values of $f_{1}$, i.e., $P \in \Sigma_{1}$ if and only if $f_{1}^{-1}(P)$ is a singular variety. Then, for any $P \in Y_{1} \backslash \Sigma_{1}, f_{1}^{-1}(P)$ is a smooth rational curve.

(3) $\Sigma_{1} \neq \emptyset$, and for any $P \in \Sigma_{1}, f_{1}^{-1}(P)$ is a reduced curve consisting of two smooth rational curves $E_{P}^{1}$ and $E_{P}^{2}$ joined at one point transversally.

(4) $D_{1}$ is smooth over $k$ and $\left.f_{1}\right|_{D_{1}}: D_{1} \rightarrow Y_{1}$ is étale.

(5) $\left(E_{P}^{1} \cdot D_{1}\right)=a_{1}+1$ and $\left(E_{P}^{2} \cdot D_{1}\right)=a_{2}+1$ for any $P \in \Sigma_{1}$. Moreover, $D_{1}$ does not pass through $E_{P}^{1} \cap E_{P}^{2}$. 
(6) There is a map $\sigma: \Sigma_{1} \rightarrow\{1,2\}$ such that

$$
D_{1} \in\left|L_{1}^{\otimes a_{1}+a_{2}+2} \otimes f_{1}^{*}\left(M_{1}\right) \otimes \mathcal{O}_{X_{1}}\left(-\sum_{P \in \Sigma_{1}}\left(a_{\sigma(P)}+1\right) E_{P}^{\sigma(P)}\right)\right| .
$$

(7) $\operatorname{deg}\left(M_{1}\right)$ is divisible by $\left(a_{1}+1\right)\left(a_{2}+1\right)$.

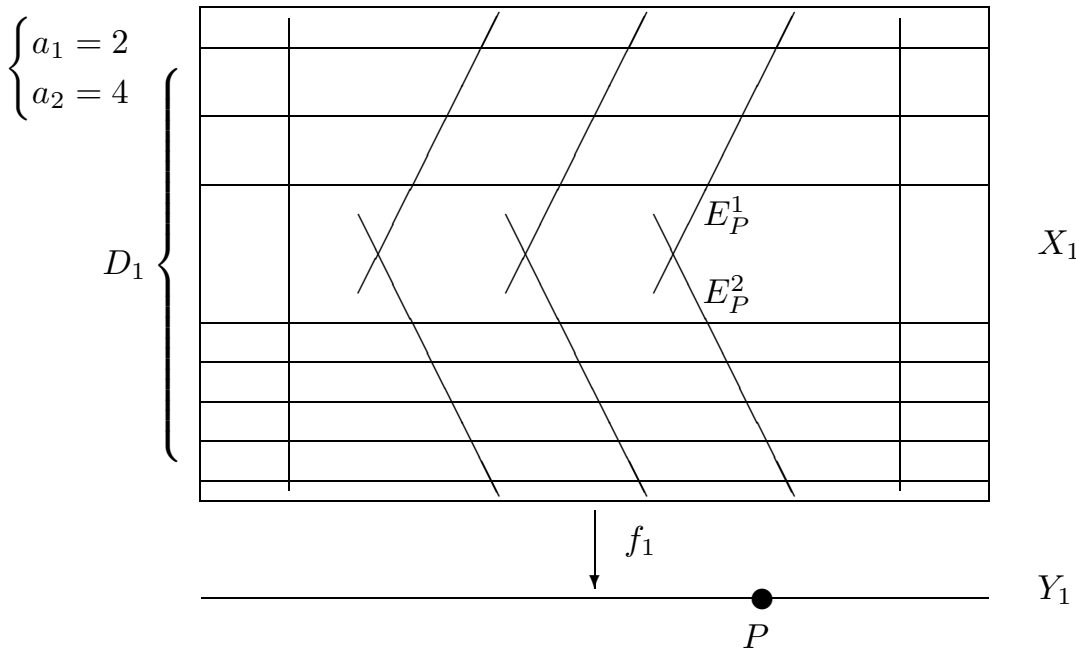

Proof. First of all, let us consider the function $\theta(x)$ defined by

$$
\theta(x)=\left(a_{1}+a_{2}+1\right) \int_{0}^{x} t^{a_{1}}(t-1)^{a_{2}} d t .
$$

Then, $\theta(x)$ is a monic polynomial of degree $a_{1}+a_{2}+1$ over $\mathbb{Q}$. Moreover, it is easy to see that

$$
\begin{gathered}
\theta^{\prime}(x)=\left(a_{1}+a_{2}+1\right) x^{a_{1}}(x-1)^{a_{2}}, \\
\theta(0)=0 \text { and } \theta(1)=(-1)^{a_{2}}\left(a_{1}+a_{2}+1\right) \frac{\left(a_{1}\right) !\left(a_{2}\right) !}{\left(a_{1}+a_{2}\right) !} .
\end{gathered}
$$

Thus, there are distinct non-zero algebraic numbers $\alpha_{1}, \ldots, \alpha_{a_{2}}$ and $\beta_{1}, \ldots, \beta_{a_{1}}$ such that

$$
\theta(x)=x^{a_{1}+1}\left(x-\alpha_{1}\right) \cdots\left(x-\alpha_{a_{2}}\right)
$$

and

$$
\theta(x)-\theta(1)=(x-1)^{a_{2}+1}\left(x-1-\beta_{1}\right) \cdots\left(x-1-\beta_{a_{1}}\right) .
$$

Here we set

$$
F(X, Y)=Y^{a_{1}+a_{2}+1} \theta(X / Y)=X^{a_{1}+1}\left(X-\alpha_{1} Y\right) \cdots\left(X-\alpha_{a_{2}} Y\right)
$$

and

$$
G(X, Y, S, T)=T F(X, Y)-Y^{a_{1}+a_{2}+1} S .
$$

Then, $F$ is a homogeneous polynomial of degree $a_{1}+a_{2}+1$ over $\mathbb{Q}$, and $G$ is a bi-homogeneous polynomial of bi-degree $\left(a_{1}+a_{2}+1,1\right)$ in $\mathbb{Q}[X, Y] \otimes_{\mathbb{Q}} \mathbb{Q}[S, T]$. Let $D^{\prime}$ (resp. $D^{\prime \prime}$ ) be the curve on $\mathbb{P}_{(X, Y)}^{1} \times \mathbb{P}_{(S, T)}^{1}$ given by the equation $\{G=0\}$ (resp. $\{Y=0\})$, where $\mathbb{P}_{(X, Y)}^{1}=\operatorname{Proj}(k[X, Y])$ and $\mathbb{P}_{(S, T)}^{1}=\operatorname{Proj}(k[S, T])$. Moreover, we 
set $D=D^{\prime}+D^{\prime \prime}$. Let $p: \mathbb{P}_{(X, Y)}^{1} \times \mathbb{P}_{(S, T)}^{1} \rightarrow \mathbb{P}_{(X, Y)}^{1}$ and $q: \mathbb{P}_{(X, Y)}^{1} \times \mathbb{P}_{(S, T)}^{1} \rightarrow \mathbb{P}_{(S, T)}^{1}$ be the natural projections. Then, $D^{\prime}$ (resp. $\left.D^{\prime \prime}\right)$ is an element of the linear system $\left|p^{*}\left(\mathcal{O}_{\mathbb{P}^{1}}\left(a_{1}+a_{2}+1\right)\right) \otimes q^{*}\left(\mathcal{O}_{\mathbb{P}^{1}}(1)\right)\right|\left(\operatorname{resp}\left|p^{*}\left(\mathcal{O}_{\mathbb{P}^{1}}(1)\right)\right|\right)$. Thus,

$$
\begin{gathered}
D \in\left|p^{*}\left(\mathcal{O}_{\mathbb{P}^{1}}\left(a_{1}+a_{2}+2\right)\right) \otimes q^{*}\left(\mathcal{O}_{\mathbb{P}^{1}}(1)\right)\right|, \\
\left(D^{\prime} \cdot D^{\prime \prime}\right)=1 \quad \text { and } \quad D^{\prime} \cap D^{\prime \prime}=\{((1: 0),(1: 0))\} .
\end{gathered}
$$

Here we claim the following.

Claim A.1.1. (a) $D^{\prime}$ is a smooth rational curve.

(b) Let $\pi^{\prime}: D^{\prime} \rightarrow \mathbb{P}_{(S, T)}^{1}$ be the morphism induced by the projection $q: \mathbb{P}_{(X, Y)}^{1} \times$ $\mathbb{P}_{(S, T)}^{1} \rightarrow \mathbb{P}_{(S, T)}^{1}$. If we set $Q_{1}=((0: 1),(0: 1)), Q_{2}=((1: 1),(\theta(1), 1))$ and $Q_{3}=((1: 0),(1: 0))$, then the set of ramification points of $\pi^{\prime}$ is $\left\{Q_{1}, Q_{2}, Q_{3}\right\}$. Further, the ramification indices at $Q_{1}, Q_{2}$ and $Q_{3}$ are $a_{1}+1, a_{2}+1$ and $a_{1}+a_{2}+1$ respectively.

Proof. (a) Since $F(X, Y)$ has no factor of $Y$, the morphism $e: \mathbb{P}_{(X, Y)}^{1} \rightarrow D^{\prime}$ given by

$$
e(x: y)=\left((x: y),\left(F(x, y): y^{a_{1}+a_{2}+1}\right)\right)
$$

is well defined. Moreover, if we set $e^{\prime}=\left.p\right|_{D^{\prime}}$, then it is easy to see that $e \cdot e^{\prime}=\mathrm{id}_{D^{\prime}}$ and $e^{\prime} \cdot e=\mathrm{id}_{\mathbb{P}^{1}}$. Thus, $D^{\prime}$ is a smooth rational curve.

(b) Pick up a point $(\lambda: \mu) \in \mathbb{P}_{(S, T)}^{1}$. Then, $G_{(\lambda, \mu)}=\mu F(X, Y)-Y^{a_{1}+a_{2}+1} \lambda$ is a homogeneous polynomial of degree $a_{1}+a_{2}+1$.

First, we assume that $\mu \neq 0$, hence we may assume that $\mu=1$. Then, $Y$ is not a factor of $G_{(\lambda, 1)}(X, Y)$, which means that $\pi^{\prime-1}((\lambda: 1))$ sits in the affine open set $\operatorname{Spec}(k[X / Y, S / T])$. Thus,

$$
\pi^{\prime-1}((\lambda: 1))=\{((\gamma: 1),(\lambda: 1)) \mid \theta(\gamma)-\lambda=0\}
$$

Hence, in order to get ramification points of $\pi^{\prime}$, we need to see multiple roots of $\phi(x)=\theta(x)-\lambda$. Here we will check that $\phi(x)$ has a multiple root if and only if $\lambda$ is either 0 or $\theta(1)$. Moreover, if $\lambda$ is 0 (resp. $\theta(1)$ ), then 0 (resp. 1) is the only multiple root of $\phi(x)$ with multiplicity $a_{1}+1$ (resp. $\left.a_{2}+1\right)$.

Let $\gamma$ be a multiple root of $\phi(x)=0$. Then, $\phi(\gamma)=\phi^{\prime}(\gamma)=0$. Here,

$$
\phi^{\prime}(x)=\left(a_{1}+a_{2}+1\right) x^{a_{1}}(x-1)^{a_{2}} .
$$

Thus, $\gamma$ is either 0 or 1 . If $\gamma=0$, then $\lambda=\theta(0)=0$. If $\gamma=1$, then $\lambda=\theta(1)$. In the same way, we can easily check the remaining part of our assertion.

Therefore, we get two ramification points $Q_{1}$ and $Q_{2}$ whose ramification indices are $a_{1}+1$ and $a_{2}+1$ respectively.

Next, we assume that $\mu=0$, hence we may assume $\lambda=1$. Then, $G_{(\lambda, \mu)}=$ $-Y^{a_{1}+a_{2}+1}$. Thus, $P_{3}$ is a ramification point whose ramification index is $a_{1}+a_{2}+$ 1.

Here we set $P_{i}=q\left(Q_{i}\right)(i=1,2,3), b_{1}=a_{1}+1, b_{2}=a_{2}+1$, and $b_{3}=a_{1}+a_{2}+1$.

Claim A.1.2. There is a cyclic covering $h_{1}: Y_{1} \rightarrow \mathbb{P}_{(S, T)}^{1}$ of smooth projective curves such that $\operatorname{deg}\left(h_{1}\right)=b_{1} b_{2} b_{3}$ and that, for any $i=1,2,3$ and any $P \in h_{1}^{-1}\left(P_{i}\right)$, the ramification index of $h_{1}$ at $P$ is $b_{i}$. 
Proof. Since $b_{1} b_{2}+b_{2} b_{3}+b_{3} b_{1} \leq 3 b_{1} b_{2} b_{3}$, there is an effective and reduced divisor $d$ on $\mathbb{P}_{(S, T)}^{1}$ such that $P_{i} \notin \operatorname{Supp}(d)$ for each $i=1,2,3$ and

$$
b_{2} b_{3} P_{1}+b_{3} b_{1} P_{2}+b_{1} b_{2} P_{3}+d \in\left|\mathcal{O}_{\mathbb{P}^{1}}\left(3 b_{1} b_{2} b_{3}\right)\right| .
$$

Let $w$ be a section of $H^{0}\left(\mathcal{O}_{\mathbb{P}^{1}}\left(3 b_{1} b_{2} b_{3}\right)\right)$ with $\operatorname{div}(w)=b_{2} b_{3} P_{1}+b_{3} b_{1} P_{2}+b_{1} b_{2} P_{3}+d$. Then, $w$ gives rise to the ring structure on $\bigoplus_{i=0}^{b_{1} b_{2} b_{3}-1} \mathcal{O}_{\mathbb{P}^{1}}(-3 i)$. Let $Y_{1}$ be the normalization of

$$
\operatorname{Spec}\left(\bigoplus_{i=0}^{b_{1} b_{2} b_{3}-1} \mathcal{O}_{\mathbb{P}^{1}}(-3 i)\right)
$$

and $h_{1}: Y_{1} \rightarrow \mathbb{P}^{1}$ the induced morphism. Then, by our choice of $w$, it is easy to see that $h_{1}: Y_{1} \rightarrow \mathbb{P}^{1}$ satisfies the desired properties.

Let $p_{1}: \mathbb{P}_{(X, Y)}^{1} \times Y_{1} \rightarrow \mathbb{P}_{(X, Y)}^{1}$ and $q_{1}: \mathbb{P}_{(X, Y)}^{1} \times Y_{1} \rightarrow Y_{1}$ be the natural projections, and $u_{1}=\mathrm{id} \times h_{1}: \mathbb{P}_{(X, Y)}^{1} \times Y_{1} \rightarrow \mathbb{P}_{(X, Y)}^{1} \times \mathbb{P}_{(S, T)}^{1}$. Then, we have the following commutative diagram:

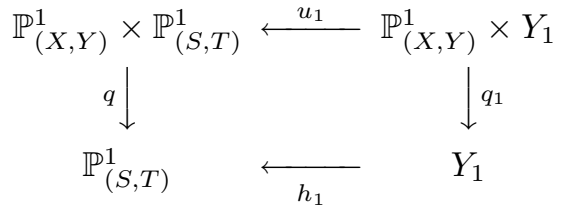

We set $h_{1}^{-1}\left(P_{1}\right), h_{1}^{-1}\left(P_{2}\right)$ and $h_{1}^{-1}\left(P_{3}\right)$ as follows:

$$
\left\{\begin{array}{l}
h_{1}^{-1}\left(P_{1}\right)=\left\{P_{1,1}, \ldots, P_{1, b_{2} b_{3}}\right\}, \\
h_{1}^{-1}\left(P_{2}\right)=\left\{P_{2,1}, \ldots, P_{1, b_{3} b_{1}}\right\}, \\
h_{1}^{-1}\left(P_{3}\right)=\left\{P_{3,1}, \ldots, P_{3, b_{1} b_{2}}\right\} .
\end{array}\right.
$$

Then, there is a unique $Q_{i, j}$ on $\mathbb{P}_{(X, Y)}^{1} \times Y_{1}$ with $q_{1}\left(Q_{i, j}\right)=P_{i, j}$ and $u_{1}\left(Q_{i, j}\right)=Q_{i}$.

Claim A.1.3. (a) $u_{1}^{*}(D)$ is étale over $Y_{1}$ outside $\left\{Q_{i, j}\right\}_{i, j}$. In particular, $u_{1}^{*}(D)$ is smooth over $k$ outside $\left\{Q_{i, j}\right\}_{i, j}$.

(b) If we set $c_{1}=a_{1}+1, c_{2}=a_{2}+1$ and $c_{3}=a_{1}+a_{2}+2$, then $u_{1}^{*}(D)$ has an ordinary $c_{i}$-fold point at $Q_{i, j}$ for every $i, j$. Moreover, each tangent of $u_{1}^{*}(D)$ at $Q_{i, j}$ is different from the fiber $q_{1}^{-1}\left(P_{i, j}\right)$.

Proof. (a) is trivial because $\left.q\right|_{D}: D \rightarrow \mathbb{P}_{(S, T)}^{1}$ is étale outside $\left\{Q_{1}, Q_{2}, Q_{3}\right\}$. Since $u_{1}^{*}\left(D^{\prime \prime}\right)=p_{1}^{-1}((1: 0))$, in order to see (b), it is sufficient to check the following. $u_{1}^{*}\left(D^{\prime}\right)$ has an ordinary $b_{i}$-fold point at $Q_{i, j}$ for every $i, j$. Moreover, for $i=1,2$, each tangent of $u_{1}^{*}\left(D^{\prime}\right)$ at $Q_{i, j}$ is different from the fiber $q_{1}^{-1}\left(P_{i, j}\right)$, and each tangent of $u_{1}^{*}\left(D^{\prime}\right)$ at $Q_{3, j}$ is different from the fiber $q_{1}^{-1}\left(P_{3, j}\right)$ and $p_{1}^{-1}((1: 0))$.

First we assume $i=1$. Let $z$ be a local parameter of $Y_{1}$ at $P_{1, j}, x=X / Y$, and $s=S / T$. Then, $(x, z)$ gives a local parameter of $\mathbb{P}_{(X, Y)}^{1} \times Y_{1}$ at $Q_{1, j}$. Since $s=v(z) z^{a_{1}+1}$ for some $v(z)$ with $v(0) \neq 0, u_{1}^{*}\left(D^{\prime}\right)$ is defined by

$$
x^{a_{1}+1}\left(x-\alpha_{1}\right) \cdots\left(x-\alpha_{a_{2}}\right)-v(z) z^{a_{1}+1}=0
$$

around $Q_{1, j}$. Thus, since $\alpha_{1} \cdots \alpha_{a_{2}} \neq 0, Q_{1, j}$ is an ordinary $\left(a_{1}+1\right)$-fold point and each tangent is different from $\{z=0\}$. 
Next we assume $i=2$. Let $z$ be a local parameter of $Y_{1}$ at $P_{2, j}, x^{\prime}=X / Y-1$, and $s^{\prime}=S / T-\theta(1)$. Then, $\left(x^{\prime}, z\right)$ gives a local parameter of $\mathbb{P}_{(X, Y)}^{1} \times Y_{1}$ at $Q_{2, j}$. Since $s^{\prime}=v(z) z^{a_{2}+1}$ for some $v(z)$ with $v(0) \neq 0, u_{1}^{*}\left(D^{\prime}\right)$ is defined by

$$
\left(x^{\prime}\right)^{a_{2}+1}\left(x^{\prime}-\beta_{1}\right) \cdots\left(x^{\prime}-\beta_{a_{1}}\right)-v(z) z^{a_{2}+1}=0
$$

around $Q_{2, j}$. Thus, we can see our assertion in this case because $\beta_{1} \cdots \beta_{a_{1}} \neq 0$.

Finally we assume that $i=3$. Let $z$ be a local parameter of $Y_{1}$ at $P_{3, j}, y=Y / X$, and $t=T / S$. Since $t=v(z) z^{a_{1}+a_{2}+1}$ for some $v(z)$ with $v(0) \neq 0, u_{1}^{*}\left(D^{\prime}\right)$ is defined by

$$
v(z) z^{a_{1}+a_{2}+1}\left(1-\alpha_{1} y\right) \cdots\left(1-\alpha_{a_{2}} y\right)-y^{a_{1}+a_{2}+1}=0
$$

around $Q_{3, j}$. Thus, $Q_{3, j}$ is an ordinary $\left(a_{1}+a_{2}+1\right)$-fold point and each tangent is different from $\{z=0\}$ and $\{y=0\}$.

Let $\mu_{1}: Z_{1} \rightarrow \mathbb{P}_{(X, Y)}^{1} \times Y_{1}$ be blowing-ups at all points $Q_{i, j}$, and let $E_{i, j}$ be a $(-1)$-curve over $Q_{i, j}$. Let $\bar{D}_{1}$ be the strict transform of $u_{1}^{*}(D)$ by $\mu_{1}$, and $g_{1}=q_{1} \cdot \mu_{1}$. Then, by the previous claim, $\bar{D}_{1}$ is étale over $Y_{1}$ and

$$
\bar{D}_{1} \in\left|\mu_{1}^{*}\left(p_{1}^{*}\left(\mathcal{O}_{\mathbb{P}^{1}}(1)\right)\right)^{\otimes a_{1}+a_{2}+2} \otimes g_{1}^{*}\left(h_{1}^{*}\left(\mathcal{O}_{\mathbb{P}^{1}}(1)\right)\right) \otimes \mathcal{O}_{Z_{1}}\left(-\sum_{i, j} c_{i} E_{i, j}\right)\right| .
$$

Let $F_{j}$ be the strict transform of the fiber $q_{1}^{-1}\left(P_{3, j}\right)$. Note that $F_{j} \cap \bar{D}_{1}=\emptyset$ for all $j$. Since the $F_{j}$ 's are $(-1)$-curves, we can contract them. Let $\nu_{1}: Z_{1} \rightarrow X_{1}$ be the contraction of the $F_{j}$ 's, and $f_{1}: X_{1} \rightarrow Y_{1}$ the induced morphism.

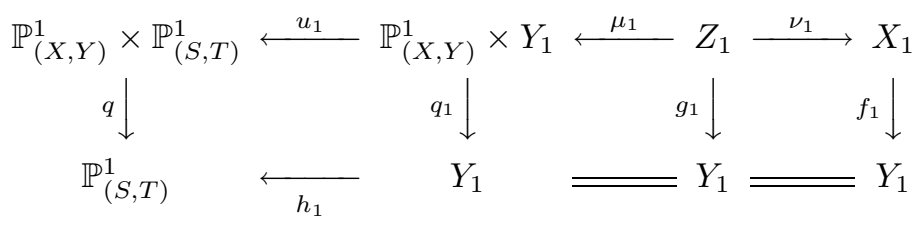

Here we set $D_{1}=\left(\nu_{1}\right)_{*}\left(\bar{D}_{1}\right), L_{1}=\left(\nu_{1}\right)_{*}\left(\mu_{1}^{*}\left(p_{1}^{*}\left(\mathcal{O}_{\mathbb{P}^{1}}(1)\right)\right)\right)^{* *}$, and

$$
M_{1}=h_{1}^{*}\left(\mathcal{O}_{\mathbb{P}^{1}}(1)\right) \otimes \mathcal{O}_{Y_{1}}\left(-\sum_{j} c_{3} P_{3, j}\right) \simeq \mathcal{O}_{Y_{1}}\left(-\sum_{j} P_{3, j}\right) .
$$

Then, since $\left(\nu_{1}\right)_{*}\left(g_{1}^{*}\left(h_{1}^{*}\left(\mathcal{O}_{\mathbb{P}^{1}}(1)\right)\right)\right)=f_{1}^{*}\left(h_{1}^{*}\left(\mathcal{O}_{\mathbb{P}^{1}}(1)\right)\right)$ and $\left(\nu_{1}\right)_{*}\left(E_{3, j}\right)=f_{1}^{*}\left(P_{3, j}\right)$ for all $j$, we can see

$$
D_{1} \in\left|L_{1}^{\otimes a_{1}+a_{2}+2} \otimes f_{1}^{*}\left(M_{1}\right) \otimes \mathcal{O}_{X_{1}}\left(-\sum_{\substack{i=1,2, j \geq 1}} b_{i} \nu_{1}\left(E_{i, j}\right)\right)\right| .
$$

Therefore, by our construction of $f_{1}: X_{1} \rightarrow Y_{1}, D_{1}, L_{1}$ and $M_{1}$, it is easy to see all properties (1)-(7) in Lemma A.1.

Proposition A.2. Let $g$ and $a$ be integers with $g \geq 1$ and $0 \leq a \leq[g / 2]$. Then, there are a smooth projective surface $X$ over $k$, a smooth projective curve $C$ over $k$, and a surjective morphism $f: X \rightarrow Y$ over $k$ with the following properties.

(1) The generic fiber of $f$ is a smooth hyperelliptic curve of genus $g$. 
(2) $f$ is not smooth and every fiber is reduced. Moreover, every singular fiber of $f$ is a nodal curve consisting of a smooth curve of genus a and a smooth curve of genus $g-a$ joined at one point.

Proof. Applying Lemma A.1 to the case where $a_{1}=2 a$ and $a_{2}=2 g-2 a$, we fix a conic fibration as in Lemma A.1. Adding one point to $\Sigma_{1}$, if necessarily, we can take an effective and reduced divisor $d$ on $Y_{1}$ such that $\Sigma_{1} \subseteq \operatorname{Supp}(d)$ and $\operatorname{deg}(d)$ is even. Thus, there is a line bundle $\vartheta$ on $Y_{1}$ with $\mathcal{O}_{Y_{1}}(d) \simeq \vartheta^{\otimes 2}$, which produces a double covering $h_{2}: Y \rightarrow Y_{1}$ of smooth projective curves such that $h_{2}$ is ramified over $\Sigma_{1}$. Let $\mu_{2}: X_{2} \rightarrow X_{1} \times_{Y_{1}} Y$ be the minimal resolution of singularities of $X_{1} \times_{Y_{1}} Y$. We set the induced morphisms as follows.

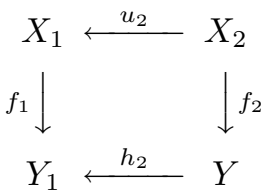

Let $\Sigma_{2}$ be the set of all critical values of $f_{2}$. Here, for all $Q \in \Sigma_{2}, f_{2}^{-1}(Q)$ is reduced, and there is the irreducible decomposition $f_{2}^{-1}(Q)=\bar{E}_{Q}^{1}+\bar{E}_{Q}^{2}+B_{Q}$ such that $u_{2}\left(\bar{E}_{Q}^{i}\right)=E_{h_{2}(Q)}^{i}$ for $i=1,2$ and $B_{Q}$ is a $(-2)$-curve. We set $D_{2}=u_{2}^{*}\left(D_{1}\right)$ and $B=\sum_{Q \in \Sigma_{2}} B_{Q}$. Then, $D_{2}$ is étale over $Y$ and $D_{2}+B$ is smooth over $k$ because $D_{2} \cap B=\emptyset$. Moreover,

$$
D_{2} \in\left|u_{2}^{*}\left(L_{1}\right)^{\otimes 2 g+2} \otimes f_{2}^{*}\left(h_{2}^{*}\left(M_{1}\right)\right) \otimes \mathcal{O}_{X_{2}}\left(-u_{2}^{*}\left(\sum_{P \in \Sigma_{1}}\left(a_{\sigma(P)}+1\right) E_{P}^{\sigma(P)}\right)\right)\right| .
$$

Let $\sigma_{2}: \Sigma_{2} \rightarrow\{1,2\}$ be the map given by $\sigma_{2}=\sigma \cdot h_{2}$. Then

$$
u_{2}^{*}\left(\sum_{P \in \Sigma_{1}}\left(a_{\sigma(P)}+1\right) E_{P}^{\sigma(P)}\right)=\sum_{Q \in \Sigma_{2}}\left(a_{\sigma_{2}(Q)}+1\right)\left(2 \bar{E}_{Q}^{\sigma_{2}(Q)}+B_{Q}\right) .
$$

Therefore,

$$
\begin{aligned}
D_{2}+B \in \mid u_{2}^{*}\left(L_{1}\right)^{\otimes 2 g+2} & \otimes f_{2}^{*}\left(h_{2}^{*}\left(M_{1}\right)\right) \\
& \otimes \mathcal{O}_{X_{2}}\left(-\sum_{Q \in \Sigma_{2}}\left(2\left(a_{\sigma_{2}(Q)}+1\right) \bar{E}_{Q}^{\sigma_{2}(Q)}+a_{\sigma_{2}(Q)} B_{Q}\right)\right) \mid
\end{aligned}
$$

Here, since $\operatorname{deg}\left(h_{2}^{*}\left(M_{1}\right)\right)=2 \operatorname{deg}\left(M_{1}\right), h_{2}^{*}\left(M_{1}\right)$ is divisible by 2 in $\operatorname{Pic}(Y)$. Further, $a_{i}$ is even for each $i=1,2$. Thus,

$$
u_{2}^{*}\left(L_{1}\right)^{\otimes 2 g+2} \otimes f_{2}^{*}\left(h_{2}^{*}\left(M_{1}\right)\right) \otimes \mathcal{O}_{X_{2}}\left(-\sum_{Q \in \Sigma_{2}}\left(2\left(a_{\sigma_{2}(Q)}+1\right) \bar{E}_{Q}^{\sigma_{2}(Q)}+a_{\sigma_{2}(Q)} B_{Q}\right)\right)
$$

is divisible by 2 in $\operatorname{Pic}\left(X_{2}\right)$, i.e., there is a line bundle $H$ on $X_{2}$ with

$$
\begin{aligned}
H^{\otimes 2} \simeq u_{2}^{*}\left(L_{1}\right)^{\otimes 2 g+2} \otimes f_{2}^{*}\left(h_{2}^{*}\left(M_{1}\right)\right) & \\
& \otimes \mathcal{O}_{X_{2}}\left(-\sum_{Q \in \Sigma_{2}}\left(2\left(a_{\sigma_{2}(Q)}+1\right) \bar{E}_{Q}^{\sigma_{2}(Q)}+a_{\sigma_{2}(Q)} B_{Q}\right)\right) .
\end{aligned}
$$


Hence, we can construct a double covering $\mu_{3}: X_{3} \rightarrow X_{2}$ of smooth projective surfaces such that $\mu_{3}$ is ramified over $D_{2}+B$. Let $f_{3}: X_{3} \rightarrow Y$ be the induced morphism. Then, there is the irreducible decomposition

$$
f^{-1}(Q)=\bar{C}_{Q}^{1}+\bar{C}_{Q}^{2}+2 \bar{B}_{Q}
$$

as cycles such that $\mu_{3}\left(\bar{C}_{Q}^{i}\right)=\bar{E}_{Q}^{i}(i=1,2)$ and $\mu_{3}\left(\bar{B}_{Q}\right)=B_{Q}$. Here it is easy to check that $\bar{B}_{Q}$ is a $(-1)$-curve. Thus, we have the contraction $\nu_{3}: X_{3} \rightarrow X$ of $\bar{B}_{Q}$ 's, and the induced morphism $f: X \rightarrow Y$.

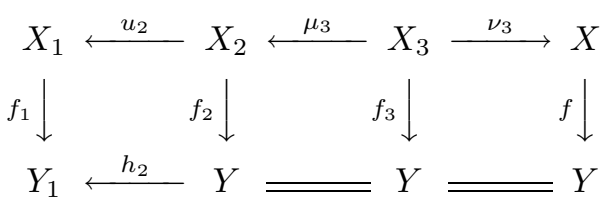

We denote $\nu_{3}\left(\bar{C}_{Q}^{i}\right)$ by $C_{Q}^{i}$. Then, $C_{Q}^{1}$ (resp. $\left.C_{Q}^{2}\right)$ is a smooth projective curve of genus $a$ (resp. $g-a),\left(C_{Q}^{1} \cdot C_{Q}^{2}\right)=1$, and $f^{-1}(Q)=C_{Q}^{1}+C_{Q}^{2}$. Thus, $f: X \rightarrow Y$ is our desired fibration.

In the same way, we can also show the following proposition.

Proposition A.3. Let $g$ and $a$ be integers with $g \geq 1$ and $0 \leq a \leq[(g-1) / 2]$. Then, there are a smooth projective surface $X$ over $k$, a smooth projective curve $C$ over $k$, and a surjective morphism $f: X \rightarrow Y$ over $k$ with the following properties.

(1) The generic fiber of $f$ is a smooth hyperelliptic curve of genus $g$.

(2) $f$ is not smooth and every fiber is reduced. Moreover, every singular fiber of $f$ is a nodal curve consisting of a smooth curve of genus a and a smooth curve of genus $g-a-1$ joined at two points.

Proof. Applying Lemma A.1 to the case where $a_{1}=2 a+1$ and $a_{2}=2 g-2 a-1$, we fix a conic fibration as in Lemma A.1. In this case, $\operatorname{deg}\left(M_{1}\right)$ is even. Thus,

$$
L_{1}^{\otimes 2 g+2} \otimes f_{1}^{*}\left(M_{1}\right) \otimes \mathcal{O}_{X_{1}}\left(-\sum_{P \in \Sigma_{1}}\left(a_{\sigma(P)}+1\right) E_{P}^{\sigma(P)}\right)
$$

is divisible by 2 in $\operatorname{Pic}\left(X_{1}\right)$. Therefore, there is a double covering $\mu: X \rightarrow X_{1}$ of smooth projective surfaces such that $\mu_{3}$ is ramified over $D_{1}$. Then, the induced morphism $f: X \rightarrow Y_{1}$ is a desired fibration.

\section{REFERENCES}

[1] F. A. Bogomolov, Holomorphic tensors and vector bundles on projective varieties, Math. USSR-Izv., 13 (1978), 499-555. MR 80j:14014

[2] J.-B. Bost, Semi-stability and height of cycles, Invent. Math., 118 (1994), 223-253. MR 95j: 14024

[3] M. Cornalba and J. Harris, Divisor classes associated to families of stable varieties, with application to the moduli space of curves, Ann. Scient. Ec. Norm. Sup., 21 (1988), 455-475. MR 89j:14019

[4] P. Deligne and D. Mumford, The irreducibility of the space of curves of given genus, Publ. Math. IHES, 36 (1969), 75-110. MR 41:6850

[5] W. Fulton, Intersection Theory, Ergebnisse der Mathematik und ihrer Grenzgebiete, 3. Folge, Band 2, (1984), Springer-Verlag. MR 85k:14004

[6] D. Gieseker, Stable vector bundles and the Frobenius morphism, Ann. Scient. Ec. Norm. Sup., 6(1973) 95-101. MR 48:3963 
[7] D. Gieseker, On a theorem of Bogomolov on Chern classes of stable bundles, Amer. J. Math., 101 (1979), 79-85. MR 80j: 14015

[8] R. Hartshorne, Ample vector bundles on curves, Nagoya Math. J. 43 (1971) 73-90. MR 45:1929

[9] J.-P. Jouanolou, Théorèmes de Bertini et Applications, Birkhäuser (1983). MR 86b:13007

[10] F. Knudsen and D. Mumford, The projectivity of the moduli space of stable curves, I: Preliminaries on "det" and "Div", Math. Scand. 39 (1976), 19-55. MR 55:10465

[11] M. Maruyama, The theorem of Grauer-Mulich-Spindler, Math. Ann. 255 (1981), 317-333. MR 82k: 14012

[12] Y. Miyaoka, The Chern Classes and Kodaira Dimension of a Minimal Variety, Advanced Studies in Pure Mathematics 10, 1987, Algebraic Geometry, Sendai, 1985, 449-476, Kinokuniya. MR 89k: 14022

[13] A. Moriwaki, Arithmetic Bogomolov-Gieseker's inequality, Amer. J. Math. 117 (1995), 13251347. MR 96i: 14022

[14] A. Moriwaki, Bogomolov conjecture over function fields for stable curves with only irreducible fibers, Comp. Math. 105 (1997), 125-140. CMP 97:10

[15] A. Moriwaki, Bogomolov conjecture for curves of genus 2 over function fields, J. Math. Kyoto Univ. 36 (1996), 687-695. CMP 97:11

[16] A. Moriwaki, A sharp slope inequality for general stable fibrations of curves, J. reine angew. Math. 480 (1996), 177-195. MR 97m:14029

[17] D. Mumford, Stability of projective varieties, L'Enseignement Math., 23 (1977), 39-110. MR 56:8568

[18] A. Paranjape and S. Ramanan, On the canonical ring of an algebraic curve, Algebraic Geometry and Commutative Algebra in Honor of Masayoshi NAGATA, vol. II (1987), 501-516. MR 90b:14024

[19] E. Ullmo, Positivité et discretion des points algébriques des courbes, preprint.

[20] G. Xiao, Fibered algebraic surfaces with low slope, Math. Ann., 276 (1987), 449-466. MR 88a:14046

[21] S. Zhang, Admissible pairing on a curve, Invent. Math., 112 (1993), 171-193. MR 94h:14023

Department of Mathematics, Faculty of Science, Kyoto University, Kyoto, 606-01, JAPAN

E-mail address: moriwaki@kusm.kyoto-u.ac.jp 